\title{
A Fully Implicit Model of the Three-Dimensional Thermohaline Ocean Circulation
}

\author{
Henk A. Dijkstra, ${ }^{* 1}$ Hakan Oksuzoglu, ${ }^{*}$ Fred. W. Wubs, $†$ and Eugen F. F. Botta $\dagger$ \\ * Institute for Marine and Atmospheric Research Utrecht, Utrecht University, Utrecht, The Netherlands; and \\ $\dagger$ Department of Mathematics and Computing Science, University of Groningen, Groningen, The Netherlands \\ E-mail: dijkstra@phys.uu.nl
}

Received June 22, 2000; revised August 3, 2001

\begin{abstract}
In this paper, a fully implicit numerical model of the three-dimensional thermohaline ocean circulation is presented. With this numerical model it is possible to follow branches of steady states in parameter space and monitor their linear stability. Also, transient flows can be computed allowing much larger time steps than those possible with explicit schemes. By using recently developed solvers for linear systems of equations and for generalized eigenvalue problems, results for reasonable spatial resolution can be obtained. Bifurcation diagrams and transient flows are computed for typical flows in a single hemispheric basin situation, with focus on (i) the performance of the methodology and (ii) the new type of information which can be obtained on these flows. (c) 2001 Academic Press
\end{abstract}

Key Words: stability of geophysical flows; numerical bifurcation theory; iterative linear systems solvers.

\section{INTRODUCTION}

Processes controlling the changes of the large-scale density driven component of the ocean circulation, called the thermohaline ocean circulation, take place on very large time scales since deep ocean velocities are small and mixing is slow. Typically, the equilibration time scale of temperature and salinity fields is in the order of 1000 years. Hence, if changes in the surface forcing occur, such as in the freshwater flux or heat flux, it takes a couple of thousand years to reach a new equilibrium state [36].

It is important to understand the different equilibria of the thermohaline circulation under given forcing conditions. Changes in circulation patterns affect the global climate state because of changes in the poleward heat transport [4]. Studies with simple box ocean models $[39,48]$ have indicated that several equilibria may be stable under the same forcing

${ }^{1}$ To whom correspondence should be addressed. 
conditions. Similar results were obtained for two-dimensional ocean models [8, 31, 42] and zonally integrated models $[29,52]$. In three-dimensional single hemispheric sector models, two different equilibrium flow patterns are found [45] when the strength of the freshwater flux forcing is large enough. When the amplitude of the forcing is increased even more, several types of time-dependent behavior are found. Variability on decadal, interdecadal, and centennial time scales is quite common and even so-called flushes appear, which are associated with a complete reorientation of the circulation pattern on very long times scales [50]. Multiple equilibria and sudden transitions in the thermohaline circulation have also been found in Atlantic basin models [5] and even in a globally coupled oceanatmosphere model [26]. An important issue in global climate change is what happens to the thermohaline circulation, when the atmospheric concentration of greenhouse gases, such as $\mathrm{CO}_{2}$, is increased. Some models predict a temporary decrease in strength of this circulation, while others show a total collapse [27].

Nearly all (relatively) low-resolution ocean circulation models that have been used to study the stability of the thermohaline circulation use an explicit time discretization, such as Leap-Frog or Adams-Bashforth schemes. Explicit schemes are relatively easy to implement but suffer from a substantial drawback. The time step is limited because of numerical amplification of truncation errors (numerical stability) rather than because of the temporal changes of the numerical solution. The time step becomes even more restricted as the spatial resolution increases. These properties are undesirable for studies of changes in the thermohaline circulation where integration times of at least a few thousand years are desired. To decrease the number of time steps, "false" transient methods, which allow for larger time steps in the deep ocean, are very common [6]. However, apart from the fact that this may distort the transient flow, the time steps are still orders of magnitude smaller than the desired integration time.

At the moment, long integration times are achieved only by relatively low resolution models. For example, in models using $4^{\circ}$ horizontal direction on a spherical grid, typical time steps can be taken of a few hours. In the coupled GFDL model, such a horizontal resolution has been used for climate impact studies of increased $\mathrm{CO}_{2}$ levels [27]. Although current climate models typically use $1^{\circ}$ horizontal resolution and about 20 vertical levels $[41,51]$, long integration times are so expensive that it is impossible to perform detailed parameter studies of the model behavior.

Since the approach to an equilibrium state is very slow for the three-dimensional thermohaline circulation, the use of implicit time discretization methods seems worthwhile. In implicit methods, the time step is not limited by numerical stability, but by the accuracy of the solution. The latter is in turn determined by the temporal changes in the numerical solution. However, implicit methods lead to large linear systems of equations, which are often ill-conditioned and hence troublesome to solve. For two-dimensional problems, direct solvers may be used but soon memory limitation boundaries are hit for three-dimensional problems. Iterative methods are needed to solve these linear systems of equations.

Implicit techniques are strongly related to the ability to solve the steady equations directly without using any time-marching techniques. The computation of steady solutions in parameter space is of interest because different regimes of behavior, for example a regime of multiple solutions, can be determined systematically. This is usually done with so-called continuation techniques combined with a Newton-Raphson-like process. When an efficient eigenvalue solver is also available, the linear stability of these steady states can be determined 
simultaneously. Methods to perform these type of computations were presented in [13] and applied to the two-dimensional Rayleigh-Benard problem. However, with these methods it is still difficult to handle three-dimensional flows, although some specific problems could be solved [10].

Semi-implicit methods are being used (e.g., in the LSG model [25] and in the POP model [14]), but fully implicit large-scale three-dimensional ocean models have not been developed so far. In our opinion, the breakthrough to realize long time scale high-resolution simulations of the ocean must come from a combination of fully implicit and explicit time-discretization techniques. Both are needed to handle both small and large time scale variability separately. The implicit transient method can be run at lower resolution and is used to compute the envelope of the fast transients, which develops on long time scales. Within this envelope, the fast time scale variability, which can only be determined at very high resolution, is then computed with explicit methods.

A first step toward this goal is the ability to handle coarse resolution ocean models with implicit methods. In this paper, we present the techniques to do this and give an impression of their performance. For the latter, we apply them to a low-resolution three-dimensional model of the thermohaline ocean circulation in a single-hemispheric basin, representing the North Atlantic. The formulation and implementation details of this model are given in Section 2. The techniques to (i) compute steady solutions in parameter space, to (ii) determine the linear stability of a steady state, and to (iii) monitor transient flows over long time scales are presented in Section 3. In the Sections 4 to 6, steady thermohaline flows, their linear stability, and their typical temporal behavior are presented. Focus is on the capabilities of the numerical techniques and the new information obtained on the flows.

\section{THE OCEAN MODEL}

In this paper, we apply the techniques to be presented in Section 3 to an ocean model, which contains the basic fluid dynamics, but does not represent the ocean physics (in particular the mixing of momentum, heat, and salt) in a "state-of-the art" way. Moreover, the configuration chosen is one that has a simple geometry to avoid additional complexities introduced by continental geometry and bottom topography. In a way, this is the first step in the development of these types of implicit ocean models; in the discussion we will comment on prospects of handling additional details.

\subsection{Governing Equations}

Consider a sector flow domain $\left[\phi_{W}, \phi_{E}\right] \times\left[\theta_{S}, \theta_{N}\right]$ representing an ocean basin on a sphere with radius $r_{0}$. The basin is rotating with angular velocity $\Omega=\eta_{f} \Omega_{0}$ and has constant depth $D$. The ocean velocities in eastward and northward directions are indicated by $u$ and $v$, the vertical velocity is indicated by $w$, the pressure by $p$, and the temperature and salinity by $T$ and $S$, respectively. Vertical and horizontal mixing of momentum and of heat and salt is represented by eddy diffusivities with horizontal and vertical friction coefficients $A_{H}$ and $A_{V}$ for momentum, and horizontal and vertical diffusivities $K_{H}$ and $K_{V}$ for heat. The mixing coefficients of salt are taken equal to those of heat. A linear equation of state is assumed with expansion coefficients $\alpha_{T}$ and $\alpha_{S}$, reference temperature $T_{0}$, salinity $S_{0}$, and density $\rho_{0}$. The governing equations, using the shallow-layer approximation $D / r_{0} \ll 1$, 
are

$$
\begin{gathered}
\frac{D u}{d t}-\frac{u v \tan \theta}{r_{0}}-2 \eta_{f} \Omega_{0} v \sin \theta \\
=-\frac{1}{\rho_{0} r_{0} \cos \theta} \frac{\partial p}{\partial \phi}+A_{V} \frac{\partial^{2} u}{\partial z^{2}}+A_{H}\left(\nabla_{H}^{2} u-\frac{u}{r_{0}^{2} \cos ^{2} \theta}-\frac{2 \sin \theta}{r_{0}^{2} \cos ^{2} \theta} \frac{\partial v}{\partial \phi}\right) \\
\frac{D v}{d t}+\frac{u^{2} \tan \theta}{r_{0}}+2 \eta_{f} \Omega_{0} u \sin \theta \\
=-\frac{1}{\rho_{0} r_{0}} \frac{\partial p}{\partial \theta}+A_{V} \frac{\partial^{2} v}{\partial z^{2}}+A_{H}\left(\nabla_{H}^{2} v-\frac{v}{r_{0}^{2} \cos ^{2} \theta}+\frac{2 \sin \theta}{\left.r_{0}^{2} \cos ^{2} \theta \frac{\partial u}{\partial \phi}\right)}\right. \\
0=\frac{\partial p}{\partial z}+\frac{1}{r_{0} \cos \theta}\left(\frac{\partial u}{\partial \phi}+\frac{\partial(v \cos \theta)}{\partial \theta}\right) \\
\frac{D T}{d t}=\nabla_{H} \cdot\left(K_{H} \nabla_{H} T\right)+\frac{\partial}{\partial z}\left(K_{V} \frac{\partial T}{\partial z}\right) \\
\frac{D S}{d t}=\nabla_{H} \cdot\left(K_{H} \nabla_{H} S\right)+\frac{\partial}{\partial z}\left(K_{V} \frac{\partial S}{\partial z}\right) \\
\rho=\rho_{0}\left(1-\alpha_{T}\left(T-T_{0}\right)+\alpha_{S}\left(S-S_{0}\right)\right)
\end{gathered}
$$

with

$$
\begin{aligned}
\frac{D}{d t} & =\frac{\partial}{\partial t}+\frac{u}{r_{0} \cos \theta} \frac{\partial}{\partial \phi}+\frac{v}{r_{0}} \frac{\partial}{\partial \theta}+w \frac{\partial}{\partial z} \\
\nabla_{H} \cdot\left(K_{H} \nabla_{H}\right) & =\frac{1}{r_{0}^{2} \cos \theta}\left[\frac{\partial}{\partial \phi}\left(\frac{K_{H}}{\cos \theta} \frac{\partial}{\partial \phi}\right)+\frac{\partial}{\partial \theta}\left(K_{H} \cos \theta \frac{\partial}{\partial \theta}\right)\right] \\
\nabla_{H}^{2} & =\nabla_{H} \cdot \nabla_{H} .
\end{aligned}
$$

The ocean circulation is driven by a wind stress $\vec{\tau}(\phi, \theta)=\tau_{0}\left(\tau^{\phi}, \tau^{\theta}\right)$, where $\tau_{0}$ is the amplitude and $\left(\tau^{\phi}, \tau^{\theta}\right)$ provides the spatial pattern. The thermohaline component of the circulation is driven by heat and freshwater fluxes at the surface. The downward heat flux $Q_{o a}$ is assumed proportional to the temperature difference between the ocean surface temperature and a prescribed atmospheric temperature $T_{S}$, i.e., $Q_{o a}=B_{T}\left(\eta_{T} T_{S}-T\right)$, with $B_{T}$ being the interfacial exchange coefficient of heat [20], and the dimensionless parameter $\eta_{T}$ is introduced to control the amplitude of $T_{S}$. The freshwater flux is converted to an equivalent salt flux and is simply a prescribed dimensionless function $F_{S}$ with amplitude $F_{0}$. At the ocean-atmosphere surface, the boundary conditions then become

$$
\begin{aligned}
\rho_{0} A_{V} \frac{\partial u}{\partial z} & =\tau_{0} \tau^{\phi} ; \quad \rho_{0} A_{V} \frac{\partial v}{\partial z}=\tau_{0} \tau^{\theta} ; \quad w=0 \\
K_{V} \frac{\partial T}{\partial z} & =B_{T}\left(\eta_{T} T_{S}-T\right) ; \quad K_{V} \frac{\partial S}{\partial z}=F_{0} F_{S} .
\end{aligned}
$$

The transfer of heat, freshwater, and momentum from the surface downward occurs in thin boundary layers, i.e., the Ekman layer for momentum transfer. Although this may be 
explicitly resolved [24], we follow the methodology applied in many low resolution ocean general circulation models. Here, the surface forcing is distributed as a body forcing over a certain depth of the upper ocean using a vertical profile function $g(z)$. More explicitly, the right-hand side of the horizontal momentum (1a) and (1b), temperature (1e), and salinity (1f) equations are extended with the source terms

$$
\begin{aligned}
Q_{\tau}^{\phi} & =g(z) \frac{\tau_{0}}{\rho_{0} H_{m}} \tau^{\phi} ; & Q_{\tau}^{\theta}=g(z) \frac{\tau_{0}}{\rho_{0} H_{m}} \tau^{\theta} \\
Q_{T} & =g(z) \frac{\eta_{T} T_{S}-T}{\tau_{T}} ; & Q_{S}=g(z) \frac{F_{0}}{H_{m}} F_{S},
\end{aligned}
$$

where $H_{m}$ is a typical vertical scale of variation of the function $g(z)$ and $\tau_{T}$ is a restoring time scale to the atmospheric forcing. Using these source terms, the boundary conditions for temperature, salinity, and wind stress at the ocean-atmosphere boundary are changed into no-flux conditions. This guarantees, for example, that the surface integral of the heat flux is zero for each steady solution [46].

A nondimensional temperature $\hat{T}$, salinity $\hat{S}$, and pressure $\hat{p}$ are introduced through $T=T_{0}+\Delta T \hat{T}, S=S_{0}+\Delta S \hat{S}$ and $p=-\rho_{0} g z+2 \Omega_{0} r_{0} U \rho_{0} \hat{p}$, where a characteristic horizontal velocity is indicated by $U$. The governing equations are further nondimensionalized using scales $r_{0}, D, U, D U / r_{0}, r_{0} / U$, and $\tau_{0}$ for horizontal length, vertical length, horizontal velocity, vertical velocity, time, and wind stress, respectively and become

$$
\begin{gathered}
\epsilon_{R}\left(\frac{D u}{d t}-u v \tan \theta\right)-\eta_{f} v \sin \theta \\
=-\frac{1}{\cos \theta} \frac{\partial p}{\partial \phi}+E_{V} \frac{\partial^{2} u}{\partial z^{2}}+\alpha_{\tau} g(z) \tau^{\phi}+E_{H}\left(\nabla^{2} u-\frac{u}{\cos ^{2} \theta}-\frac{2 \sin \theta}{\cos ^{2} \theta} \frac{\partial v}{\partial \phi}\right) \\
\epsilon_{R}\left(\frac{D v}{d t}+u^{2} \tan \theta\right)+\eta_{f} u \sin \theta \\
=-\frac{\partial p}{\partial \theta}+E_{V} \frac{\partial^{2} v}{\partial z^{2}}+\alpha_{\tau} g(z) \tau^{\theta}+E_{H}\left(\nabla^{2} v-\frac{v}{\cos ^{2} \theta}+\frac{2 \sin \theta}{\cos ^{2} \theta} \frac{\partial u}{\partial \phi}\right) \\
\quad 0=\frac{\partial p}{\partial z}=\frac{1}{\partial z}\left(\frac{\partial u}{\cos \theta}+\frac{\partial(v \cos \theta)}{\partial \theta}\right) \\
\frac{D T}{d t}=\nabla_{H} \cdot\left(P_{H} \nabla_{H} T\right)+\frac{\partial}{\partial z}\left(P_{V} \frac{\partial T}{\partial z}\right)+B\left(\eta_{T} T_{S}-T\right) g(z) \\
\frac{D S}{d t}=\nabla_{H} \cdot\left(P_{H} \nabla_{H} S\right)+\frac{\partial}{\partial z}\left(P_{V} \frac{\partial S}{\partial z}\right)+\gamma F_{S} g(z),
\end{gathered}
$$

where the hats are dropped for convenience. On the lateral walls, slip conditions are prescribed to allow for two-dimensional solutions in particular cases, and the heat and salt fluxes are zero. The bottom of the ocean $(z=-1)$ is assumed to be flat, isolated, and 
impermeable to salt. The nondimensional boundary conditions are hence formulated as

$$
\begin{gathered}
z=0,-1: \frac{\partial u}{\partial z}=\frac{\partial v}{\partial z}=w=\frac{\partial T}{\partial z}=\frac{\partial S}{\partial z}=0 \\
\phi=\phi_{W}, \phi_{E}: u=\frac{\partial v}{\partial \phi}=\frac{\partial w}{\partial \phi}=\frac{\partial T}{\partial \phi}=\frac{\partial S}{\partial \phi}=0 \\
\phi=\theta_{S}, \theta_{N}: \frac{\partial u}{\partial \theta}=v=\frac{\partial w}{\partial \theta}=\frac{\partial T}{\partial \theta}=\frac{\partial S}{\partial \theta}=0 .
\end{gathered}
$$

The parameters in these equations are the Rossby number $\epsilon_{R}$, the Rayleigh number $R a$, the vertical and horizontal Ekman number $E_{V}$ and $E_{H}$, the wind stress coefficient $\alpha_{\tau}$, the vertical and horizontal inverse Peclet numbers $P_{V}$ and $P_{H}$, the Biot number $B$, and the freshwater flux strength $\gamma$. Expressions for these parameters are

$$
\begin{gathered}
\epsilon_{R}=\frac{U}{2 \Omega_{0} r_{0}} ; \quad R a=\frac{\alpha_{T} \Delta T g D}{2 \Omega_{0} U r_{0}} ; \quad E_{V}=\frac{A_{V}}{2 \Omega_{0} D^{2}} ; \quad E_{H}=\frac{A_{H}}{2 \Omega_{0} r_{0}^{2}} ; \quad \alpha_{\tau}=\frac{\tau_{0}}{2 \Omega_{0} \rho_{0} H_{m} U} \\
\lambda=\frac{\alpha_{S} \Delta S}{\alpha_{T} \Delta T} ; \quad P_{H}=\frac{K_{H}}{U r_{0}} ; \quad P_{V}=\frac{K_{V} r_{0}}{U D^{2}} ; \quad B=\frac{r_{0}}{U \tau_{T}} ; \quad \gamma=\frac{F_{0} r_{0}}{U \Delta S H_{m}} .
\end{gathered}
$$

Apart from parameters in the forcing functions, such as $\eta_{T}$ and the dimensionless parameter $\eta_{f}$, which will be used to follow solutions continuously between nonrotating and rotating cases, the system appears to contain 10 parameters. However, only 8 of these are independent; when the salt field is rescaled with a factor $\lambda$, the product $\lambda \gamma$ appears, which is an independent parameter. Moreover, the characteristic velocity $U$ can be chosen as a function of other parameters, reducing the number of parameters again by one.

Given the surface wind stress $\vec{\tau}$, the atmospheric temperature $T_{S}$, the freshwater flux $F_{S}$, and values of the dimensionless parameters, the time-evolution from a particular initial condition is well defined.

\subsection{Convective Adjustment}

Since convection, which occurs in case of an unstable stratification, is not resolved by the hydrostatic model, an explicit representation is needed to obtain stably stratified solutions. A first variant of convective adjustment used in the model is local implicit mixing [53]. This means that when the flow becomes unstably stratified, the vertical mixing coefficient of heat and salt is increased according to

$$
P_{V}=P_{V}^{0}+P_{V}^{c} \mathcal{H}\left(\lambda \frac{\partial S}{\partial z}-\frac{\partial T}{\partial z} ; \epsilon_{H}\right),
$$

where $P_{V}^{0}$ is the background inverse Peclet number, $P_{V}^{c}$ is the convective inverse Peclet number which is much larger than $P_{V}^{0}$, and $\mathcal{H}$ is a continuous approximation to the Heaviside function. For the latter we use

$$
\mathcal{H}\left(x ; \epsilon_{H}\right)=\frac{1}{2}\left(1+\tanh \frac{x}{\epsilon_{H}}\right),
$$

where $\epsilon_{H}=0.1$. The ratio of mixing coefficients is monitored by a dimensionless parameter $C_{a}=P_{V}^{c} / P_{V}^{0}$, and only $C_{a} \rightarrow \infty$ guarantees a stable stratification. 
A second variant used, developed in [47], is called the global adjustment procedure (GAP). The GAP starts off with the unstably stratified solution, say $\mathbf{u}^{1}=\mathbf{u}$, and a constant field of vertical diffusivity $P_{V}^{1}(\phi, \theta, z)=P_{V}^{0}$, with $P_{V}^{0}$ the standard value of vertical diffusivity. Within a step $k$ of an iterative loop over $N_{a}$ steps, a stably stratified solution $\mathbf{u}_{s t}^{k}$ is constructed from $\mathbf{u}^{k}$, using the convective adjustment procedure of [32]. A linear combination is taken,

$$
\tilde{\mathbf{u}}^{k}=\left(1-\omega_{k}\right) \mathbf{u}^{k}+\omega_{k} \mathbf{u}_{s t}^{k} \quad k=1, N_{a}
$$

where $\omega_{k}$ increases from zero to unity in $N_{a}$ steps (according to $\omega_{k}=\frac{1}{2}\left(1-\cos \pi k / N_{a}\right)$ ). The vertical diffusivities are adjusted according to

$$
P_{V}^{k+1}=P_{V}^{c} \tanh \left(\Gamma \frac{P_{V}^{k}}{P_{V}^{c}}\right) ; \quad \Gamma \equiv \frac{\partial \rho^{k} / \partial z}{\partial \tilde{\rho}^{k} / \partial z},
$$

where $P_{V}^{c}$ is an upper bound on the vertical diffusivities. In regions that are stabilized by a pass of the adjustment procedure, $\Gamma>1$. For modest changes in the stratification (e.g., when $\omega_{k}$ is still small), this procedure guarantees that the vertical diffusive fluxes of buoyancy associated with $\tilde{\mathbf{u}}$ and $\mathbf{u}$ are the same. For large values of $\Gamma$ (i.e., in well-mixed areas when $\omega_{k} \rightarrow 1$ ), the vertical diffusivity is bounded by $P_{V}^{c}$. A Newton step (Section 3 ) is performed on $\tilde{\mathbf{u}}^{k}$ to obtain a new estimate $\mathbf{u}^{k+1}$, which includes an update of the velocity field. After $N_{a}$ steps, the procedure is repeated with $\omega_{k}=1$ for $k>N_{a}$ until convergence is reached. The resulting solution $\mathbf{u}_{s t}$ is stably stratified, has enhanced diffusivities in the regions where convection took place, and has a velocity field that is consistent with the density field.

\section{NUMERICAL METHODS}

The equations are discretized in space using a second-order accurate control volume discretization method on a staggered (Marker and Cell or Arakawa C-) grid with $i=$ $0, \ldots, N, j=0, \ldots, M, k=0, \ldots, L$. The unknowns are labeled from left to right, from south to north, and from bottom to top, with $z_{0}=-1$ and $z_{L}=0$. Here, the $p, T$, and $S$ points are in the center and the $u, v$, and $w$ points are on the cell boundaries. The function $g(z)$, appearing in (3), is chosen as

$$
g(z)=\mathcal{H}\left(z-z_{L-1}, \epsilon_{H}\right)
$$

with again $\mathcal{H}$ being the Heaviside function (7) with $\epsilon_{H}=10^{-6}$. In this way, the input of each quantity through the ocean-atmosphere surface (zonal and meridional momentum, heat, and salt) is distributed as a source term over the most upper level. The spatially discretized model equations can be written in the form

$$
\mathbf{M} \frac{d \mathbf{u}}{d t}=\mathbf{F}(\mathbf{u})=\mathbf{L}(\mathbf{u})+\mathbf{N}(\mathbf{u}, \mathbf{u})
$$

where the vector $\mathbf{u}$ contains the unknowns $(u, v, w, p, T, S)$ at each grid point and hence has dimension $d=6 \times N \times M \times L$. The operators $\mathbf{M}$ and $\mathbf{L}$ are linear and $\mathbf{N}$ represents the nonlinear terms in the equations. 


\subsection{Continuation of Steady States}

Steady-state solutions lead to a set of nonlinear algebraic equations of the form

$$
\mathbf{F}(\mathbf{u}, \mathbf{p})=0 .
$$

Here the parameter dependence of the equations is made explicit through the $p$-dimensional vector of parameters $\mathbf{p}$ and hence $\mathbf{F}$ is a nonlinear mapping from $\mathbf{R}^{d+p} \rightarrow \mathbf{R}^{d}$.

As can be readily seen from the continuous form of the steady equations, the salinity is determined up to an additive constant. Moreover, as is in the general problem, also the pressure is determined up to an additive constant. To calculate a steady-state solution of the system of equations, the equations are regularized (such that the Jacobian matrix is nonsingular at each regular point) by fixing the pressure at a particular point (in our case at the point $(N, M, L))$. In addition, an integral condition for $S$ is substituted for the last equation from the salinity equation, such that salt is conserved exactly within the domain. Since the total dimensional salt content is $\rho_{0} V S_{0}$, where $V$ is the total volume of the basin, the scaling for salinity provides the dimensionless form as

$$
\int_{V} S \cos \theta d \phi d \theta d z=0,
$$

which is a constraint on the deviation of the salinity field from uniform conditions. To determine branches of steady solutions of the (now slightly modified) equations (12) as one of the parameters, say $\mu$, is varied, the pseudo-arclength method [22] is used. The branches $(\mathbf{u}(s), \mu(s))$ are parameterized by an "arclength" parameter $s$. An additional equation is obtained by "normalizing" the tangent

$$
\dot{\mathbf{u}}_{0}^{T}\left(\mathbf{u}-\mathbf{u}_{0}\right)+\dot{\mu}_{0}\left(\mu-\mu_{0}\right)-\Delta s=0,
$$

where $\left(\mathbf{u}_{0}, \mu_{0}\right)$ is an analytically known starting solution or a previously computed point on a particular branch and $\Delta s$ is the step-length.

To solve the system of equations (12-14), Euler-Newton continuation is used. The $(d+$ $1) \times(d+1)$ Jacobian matrix $\mathcal{J}(s)$ of $(12-14)$ along a branch is given by

$$
\mathcal{J}(s)=\left[\begin{array}{cc}
\Phi & \mathbf{F}_{\mu} \\
\dot{\mathbf{u}}_{0}^{T} & \dot{\mu}_{0}
\end{array}\right],
$$

where $\Phi$ is the matrix of derivatives of $\mathbf{F}$ to $\mathbf{u}$ and $\mathbf{F}_{\mu}$ is the derivative to the parameter $\mu$. During one Newton iteration, linear systems of the form

$$
\mathcal{J}\left(\begin{array}{c}
\Delta \mathbf{u} \\
\Delta \mu
\end{array}\right)=\left(\begin{array}{c}
\mathbf{r} \\
r_{d+1}
\end{array}\right)
$$

have to be solved, where $\Delta \mathbf{u}$ and $\Delta \mu$ are updates during the Newton process and $\mathbf{r}$ and $r_{d+1}$ derive from the right-hand sides of (12) and (14).

One can split the solution of (16) into two steps in which only linear systems with $\Phi$ are solved. If $\mathbf{z}_{1}$ and $\mathbf{z}_{2}$ are solved from

$$
\begin{aligned}
& \Phi \mathbf{z}_{1}=\mathbf{r} \\
& \Phi \mathbf{z}_{2}=\mathbf{F}_{\mu},
\end{aligned}
$$


then the solution $(\Delta \mathbf{u}, \Delta \mu)$ is found from

$$
\begin{aligned}
\Delta \mu & =\frac{r_{d+1}-\dot{\mathbf{u}}_{0}^{T} \mathbf{z}_{1}}{\dot{\mu}_{0}-\dot{\mathbf{u}}_{0}^{T} \mathbf{z}_{2}} \\
\Delta \mathbf{u} & =\mathbf{z}_{1}-\Delta \mu \mathbf{z}_{2} .
\end{aligned}
$$

\subsection{Stability of Steady States}

When a steady state is determined, the linear stability of the solution is considered and transitions that mark qualitative changes, such as transitions to multiple equilibria (pitchfork bifurcations of limit points) or periodic behavior (Hopf bifurcations), can be detected. The linear stability analysis amounts to solving a generalized eigenvalue problem of the form

$$
\mathcal{A} \mathbf{x}=\sigma \mathcal{B} \mathbf{x}
$$

where $\mathcal{A}=\Phi$ and $\mathcal{B}=-\mathbf{M}$ are in general nonsymmetric matrices. If $\mathcal{B}$ is nonsingular, the problem reduces to an ordinary eigenvalue problem for the matrix $\mathcal{B}^{-1} \mathcal{A}$. Because only real matrices are considered, there are $d$ eigenvalues, which are either real or occur as complex conjugate pairs. However, if $\mathcal{B}$ is singular, the eigenvalue structure may be more complicated; the set of eigenvalues may be finite, empty, or even the whole complex plane [17]. In the particular model here, $\mathcal{B}$ is a singular diagonal matrix because time derivatives are absent in the continuity equation and vertical momentum equation.

Traditional eigenvalue solvers (e.g., the QZ algorithm [17]) which determine all eigenvalues and, if desired, all eigenvectors are impossible to use. However, in many hydrodynamic stability problems, the instability of a certain steady flow pattern occurs only through a small number of modes and one is only interested to compute a few eigenmodes, i.e., those with eigenvalues closest to the imaginary axis (the "most dangerous" modes). Goldhirsch et al. [16] present three different versions of an algorithm to determine only a few of these most dangerous modes suited for nonsymmetric eigenvalue problems. In [9], a combination of spectral transformations and the Arnoldi algorithm [33] is used and applied to determine the linear stability of steady (coating) flows. A variant of the methods in [9] was used in [13], being a combination of a spectral transformation and the simultaneous iteration technique [38]. As in [9], the idea of the algorithm is to transform the eigenvalue problem in such a way that the most dangerous modes become the most dominant modes (i.e., those with eigenvalues of largest norm). In this way, generalized power methods can be used on the transformed problem.

A new method to determine eigensolutions of large sparse generalized eigenvalue problems is the Jacobi-Davidson QZ-method (JDQZ) [37]. Using this method, one can compute several, say $m$, eigenvalues and optionally eigenvectors of the generalized eigenvalue problem

$$
\beta \mathcal{A q}=\alpha \mathcal{B} \mathbf{q}
$$

where $\mathcal{A}, \mathcal{B}$ are matrices with complex entries and $\alpha$ and $\beta$ are complex numbers. The pair $(\alpha, \beta)$ is called an eigenvalue with corresponding eigenvector $\mathbf{q}$. In each step of the Jacobi-Davidson method, a search space $V$ and a test space $W$ are constructed and a new approximation $\tilde{q}$ of the eigenvector is selected from a search space $V$, together with a 
new approximation of the eigenvalue near a chosen target $\tau$. The details of the method are described elsewhere [37] and the implementation of JDQZ is described in an earlier version of our continuation code in [43].

\subsection{Implicit Time Integration}

A nice spin-off of steady-state solvers is the immediate availability of an implicit time integration scheme. Using a time step $\Delta t$, and a time index $n$, a class of two-level schemes can be written as

$$
\mathbf{M} \frac{\mathbf{u}^{n+1}-\mathbf{u}^{n}}{\Delta t}+\Theta \mathbf{F}\left(\mathbf{u}^{n+1}\right)+(1-\Theta) \mathbf{F}\left(\mathbf{u}^{n}\right)=0 .
$$

For $\Theta=1$, this is the Backward Euler scheme and for $\Theta=1 / 2$, it is the Crank-Nicholson scheme. The equations for $\mathbf{u}^{n+1}$ are solved by the Newton-Raphson technique and lead to large systems of nonlinear algebraic equations, similar to that for the steady-state computation. Note that within the time-dependent ocean model, the explicit integral condition for the salinity equations can be omitted, because the total salinity is fixed by the initial conditions.

It is well known that the Crank-Nicholson scheme is unconditionally stable for linear equations. This does not mean that one can take any time step, since this quantity is still constrained by accuracy of the solution. Although the scheme is second-order accurate in time, large discretization errors occur when the time step is too large. Another limitation on the time step is the convergence domain of the Newton-Raphson process, which does not necessarily converge for every time step. It will turn out that for the ocean model, despite these limitations, much larger time steps can be taken than with an explicit time discretization.

\subsection{Linear System Solvers}

The linear sparse matrix solver which makes these computations possible is called matrix renumbering incomplete LU (MRILU). In the next section, the method will be briefly outlined, followed by a section on the performance of the method on a typical case encountered during steady-state computation in the ocean model.

\subsubsection{Outline of the MRILU Method}

MRILU consists of a multilevel preconditioner combined with a modern conjugate gradient type iterative method such as the BICGSTAB or the GMRES (Generalized Minimal RESidual) method [2]. As a preconditioning matrix, an incomplete factorization is constructed of which the basic steps are outlined in Fig. 1. During the first step of the factorization (Step 1 in Fig. 1), a nearly independent set of unknowns is determined. For sparse matrices this set has always more than one element, but to find the maximum set is an NP-complete problem. However, in our applications the fill per equation changes little and with a simple recursive greedy algorithm, already sets close to the optimum are obtained. After the dropping of nondiagonal elements, this step yields a diagonal matrix $\tilde{A}_{11}$ as an approximation of $A_{11}$ (Step 2 in Fig. 1.) Because the inverse of this matrix is also diagonal and since $A_{12}$ and $A_{21}$ are also sparse (even made sparser by dropping small elements during Step 3), the Schur-complement computed in Step 4 will also be sparse and the process can be repeated. 
$\operatorname{Set} A^{(0)}=A$

for $\mathrm{i}=1: \mathrm{M}$

1. Make a reordering and partitioning of $A^{(i-1)}$

$$
\left[\begin{array}{ll}
A_{11} & A_{12} \\
A_{21} & A_{22}
\end{array}\right]
$$

such that the matrix $A_{11}$ is sufficiently diagonal dominant.

2. Approximate $A_{11}$ by a diagonal matrix $\tilde{A}_{11}$.

3. Drop small elements in $A_{12}$ and $A_{21}$.

4. Make an incomplete $L U$ factorization

$$
\left[\begin{array}{cc}
I & 0 \\
\tilde{A}_{21} \tilde{A}_{11}^{-1} & I
\end{array}\right]\left[\begin{array}{cc}
\tilde{A}_{11} & \tilde{A}_{12} \\
0 & A^{(i)}
\end{array}\right]
$$

where $A^{(i)}=A_{22}-\tilde{A}_{21} \tilde{A}_{11}^{-1} \tilde{A}_{12}$ (Schur complement of $\tilde{A}_{11}$ ).

end

Make an exact (or accurate incomplete) factorization of $A^{(M)}$.

FIG. 1. The basic steps of the MRILU algorithm used to solve the linear systems of equations.

During the factorization process, the fill increases and dropping is needed to get large independent sets. The dropping strategy used in Steps 2 and 3 is based on the ratio of the element at hand and the diagonal element, and on the amount dropped so far in the corresponding row and column. To handle the linear systems arising from the ocean model, an extension of the MRILU algorithm for systems of partial differential equations is used. The matrix $\tilde{A}_{11}$ is now a block-diagonal matrix in which the block size corresponds to the number of unknowns per grid cell. For the ocean model, this number equals 6 . It was observed that it is beneficial to do only a few reduction steps ( $M$ small, say 5$)$ and then make an accurate incomplete factorization of $A^{(M)}$. The diagonal blocks in the $L$ and $U$ factor (Fig. 1) allow for parallelization and vectorization as is described elsewhere [3, 30].

Loosely speaking, the matrices $A^{(i)}$ can be seen as coarse grid approximations to the linear differential operator, and the L-factor in Step 4 as a restriction operator. The prolongation operator is implicit in the U-factor and hence in multigrid terminology the factorization corresponds to one V-cycle. Hence, the method is related to multigrid methods, which have the well-known property to show convergence independent of the grid. In most convectiondiffusion problems we observe grid-independent convergence with MRILU, e.g., about 300 flops per grid point are needed to gain six digits in solving Poisson's equation in 2D with a standard 5-point discretization. This is quite low considering that in this case a matrix-vector multiplication is about 10 flops per grid point (see [1] for a comparison). To obtain this grid-independent convergence behavior it is indispensable to use lumping, which means that the diagonal in the factorization is adapted such that it produces the same 
result as the original matrix when applied to a constant vector [19]. Work to obtain also grid-independent convergence for systems of partial differential equations is in progress. Meanwhile, the current version is already a substantial improvement over traditional ILU approaches.

MRILU is not a black box solver and requires a small set of parameters to be tuned for each particular problem. For a limited number of reduction steps ( $M$ small), the most critical parameter is the drop tolerance $\varepsilon$ in the ILU factorization of the last block. This parameter determines the amount of memory to be used by the preconditioner. The smaller the tolerance, the larger the fill-in but the faster the convergence. Hence, the drop tolerance $\varepsilon$ provides a trade-off between cpu and memory usage.

The performance of MRILU can be improved by an a priori scaling of the matrix. For scalar equations, MRILU is nearly independent of diagonal scaling. However, for equations arising from systems of partial differential equations, with more than one unknown per point, the situation is different. In the latter case, the dropping is greatly influenced by diagonal block scaling. Consider, for example, the matrix

$$
\left[\begin{array}{ll}
1 & \alpha \\
\beta & 0
\end{array}\right]
$$

in which the zero is mimicking the zero block arising for the pressure in the continuity equation of the incompressible Navier-Stokes equations. If $\alpha$ or $\beta$ is smaller than the drop tolerance $\varepsilon$, then the dropping of one of these causes the matrix to become singular, which is undesired. This can be avoided by scaling the equations and the unknowns such that $\alpha$ and $\beta$ are both of magnitude 1. In practice this singular case is rarely seen, but often more coefficients for one type of unknown, say those related to the pressure, are dropped than for those for another type, for example a velocity component. In general, the convergence of the final method is determined by the part in which most coefficients are dropped, making the higher fill in the other part of the matrix useless. This imbalance should be avoided in order to obtain an efficient method.

\subsubsection{Performance on the Ocean Model}

We show here timing and memory requirements of these methods to compute a typical steady ocean flow with the model presented in Section 2. The more detailed procedure to compute a next steady state $(\mathbf{u}, \mu)$ from one which has already been determined $\left(\mathbf{u}_{0}, \mu_{0}\right)$, (assuming that the tangent $\left(\dot{\mathbf{u}}_{0}, \dot{\mu}\right)$ is available) when changing a parameter $\mu$ through a choice of $\Delta s$, is as follows:

(i) Start the Newton process with initial solution $\mathbf{u}^{0}=\mathbf{u}_{0}+\Delta s \dot{\mathbf{u}}_{0}$ and $\mu^{0}=\mu_{0}+\dot{\mu} \Delta s$.

(ii) Compute the quantities $\mathbf{r}, r_{d+1}$, and the Jacobian $\mathcal{J}$ the latter in the form (15). The Jacobian is assembled from local matrices representing the differential operators on the stencil as described in [11]. The matrix $\Phi$ is stored in compressed row storage (CRS) form [34].

(iii) Solve the two systems (17) with the MRILU method. Since the matrix for both systems is the same, only one factorization is made and hence one preconditioning matrix is constructed. The GMRES or BICGSTAB iteration is stopped when the absolute residue is smaller than $10^{-6}$.

(iv) Update the solution $\mathbf{u}^{k+1}=\mathbf{u}^{k}+\Delta \mathbf{u}^{k}, \mu^{k+1}=\mu^{k}+\Delta \mu^{k}$ within the Newton iteration. 


\section{TABLE I}

Three Different Choices of Column and Row Scaling Coefficients of the Matrix $\Phi$ Appearing in (15)

\begin{tabular}{clcccccc}
\hline Scaling & & $\mathrm{u}$ & $\mathrm{v}$ & $\mathrm{w}$ & $\mathrm{p}$ & $\mathrm{T}$ & $\mathrm{S}$ \\
\hline \multirow{2}{*}{1} & Column & 1. & 1. & 100. & 1. & 100. & 10. \\
& Row & $d \phi$ & $d \theta$ & $d z$ & $d z * .01$ & .01 & .1 \\
2 & Column & 1. & 1. & 100. & 1. & 10. & 1. \\
& Row & $d \phi$ & $d \theta$ & $d z$ & $d z * .01$ & .1 & 1. \\
3 & Column & 1. & 1. & 10. & 1. & 100. & 10. \\
& Row & $d \phi$ & $d \theta$ & $d z$ & $d z * .1$ & .01 & .1 \\
\hline
\end{tabular}

(v) Repeat steps (ii) to (iv) until the Newton process converges, using a tolerance in the residue of $10^{-6}$.

The most time-consuming step is the solution of the linear systems during step (iii) above. It appears necessary to rescale the rows corresponding to the continuity, temperature, and salinity equations to get a more balanced dropping as explained above. For this purpose, also the columns associated with the vertical velocity, temperature, and salinity are rescaled. Typical scaling factors used are listed in Table I, where $d \phi, d \theta$, and $d z$ are the grid sizes in zonal, meridional, and vertical direction. There are as many scaling coefficients as the number of equations per grid point. For simplicity, the same scaling is applied to each diagonal block although this may not be the best choice. The scaling difference between choice 1 and 2 is only in the temperature and salinity equations, whereas that between 1 and 3 is in the continuity and vertical momentum equations.

As a typical case, we take the starting point $\mathbf{u}_{0}$ as the point labeled (a) in Fig. $4 \mathrm{a}$ below, $\gamma$ is chosen as the control parameter, and a step-size of $\Delta s=0.1$ is considered. The spatial resolution for this case is $20 \times 20 \times 16$, which gives 38,400 unknowns. It takes four Newton iterations to converge to the next steady solution. Table II shows the

\section{TABLE II}

The Effect of the Drop Tolerance $\varepsilon$ and the Type of Scaling (as in Table I) on the Timing of an Average Newton Step During Continuation of Steady States

\begin{tabular}{clrrrrr}
\hline Scaling & $\varepsilon\left(\times 10^{3}\right)$ & 0.6 & 1.2 & 2.4 & 4.8 & 9.6 \\
\hline \multirow{2}{*}{1} & Time (sec) & 389 & 245 & 208 & $*$ & $*$ \\
& Max. nonzero & 328 & 220 & 148 & $*$ & $*$ \\
\multirow{2}{*}{2} & Max. iteration & 20 & 33 & 55 & $*$ & $*$ \\
& Time (sec) & 299 & 232 & 365 & $*$ & $*$ \\
& Max. nonzero & 232 & 170 & 113 & $*$ & $*$ \\
3 & Max. iteration & 40 & 55 & 150 & $*$ & 513 \\
& Time (sec) & $* *$ & 723 & 340 & 253 & 124 \\
& Max. nonzero & $* *$ & 387 & 266 & 194 & 300 \\
\hline
\end{tabular}

Notes. Within the Newton step, the MRILU preconditioner is called once and the BICGSTAB method is called twice, since two linear systems with different right-hand sides for the same matrix have to be solved, according to (17). A single * entry indicates that the iterative process did not converge, while $* *$ indicates that the preconditioner required too much memory. 


\section{TABLE III}

CPU Time and Memory Used for Different Resolutions for One Newton Step Starting from Trivial Solution, with $\varepsilon=2.4 \times 10^{-3}$

\begin{tabular}{lrccc}
\hline Resolution & CPU Time & Memory Used & Unknowns & Time/unknown \\
\hline $10 \times 10 \times 16$ & $29 \mathrm{~s}$ & $122 \mathrm{MB}$ & 9,600 & $3.0 \times 10^{-3}$ \\
$20 \times 20 \times 16$ & $194 \mathrm{~s}$ & $286 \mathrm{MB}$ & 38,400 & $5.1 \times 10^{-3}$ \\
$30 \times 30 \times 16$ & $1527 \mathrm{~s}$ & $712 \mathrm{MB}$ & 86,400 & $1.7 \times 10^{-2}$ \\
\hline
\end{tabular}

effect of the drop tolerance and the different scalings on the performance of MRILU (combined with BICGSTAB) during step (iii). Maximum values were taken over the four Newton steps, and the test was done on a Compaq XP1000 500MHz workstation with $1 \mathrm{~GB}$ memory.

For each value of the drop tolerance, we have also listed the maximum number of BICGSTAB iterations and the maximum of the average number of nonzero elements per row in the incomplete LU factorization, the latter being an indicator of the memory requirements for the preconditioner. For the scaling 1, increasing the drop tolerance reduces the memory requirements while increasing the number of iterations. Increasing $\varepsilon$ by a factor 2 typically doubles the number of BICGSTAB iterations, but decreases the time for the preconditioner. Although the drop tolerance $\varepsilon=2.4 \times 10^{-3}$ gives the fastest convergence for the BICGSTAB iteration, this iteration does not converge for $\varepsilon=4.8 \times 10^{-3}$. The results also show that the performance of the linear solver is quite sensitive to the choice of scaling coefficients. When the coefficients of the vertical momentum and continuity equation are not scaled properly, the method only converges when the drop tolerance is large. When $\varepsilon$ becomes too small, too many fill-in occur and the incomplete LU factorization requires too much memory. A proper scaling of the temperature and the salinity equations can reduce the memory required (scaling 2), but this does not automatically lead to faster convergence. The results indicate that it is worthwhile to perform this type of sensitivity analysis for the method. In principle, the convergence of the Newton process does not depend on the drop tolerance, when the linear systems are solved accurately enough. However, if the BICGSTAB residue has not decreased below the desired tolerance due to a large value of the drop tolerance (for example, because only a maximum number of iterations is allowed), this can deteriorate the convergence of the Newton process. In the results shown in the next sections, we have used scaling 1 and $\varepsilon=1.2 \times 10^{-3}$.

To give an impression how the performance scales with grid size, from the starting solution $\mathbf{u}_{0}=\mu_{0}=0$, one step $\Delta s=0.1$ was taken into the direction of $\eta_{T}$. For a drop tolerance $\varepsilon=2.4 \times 10^{-3}$, the CPU-time and memory required to solve the linear systems within one Newton step is shown in Table III. Although the computational cost for the linear solver for this problem depends on the parameters chosen, it is observed that the computational cost per unknown increases substantially with the number of unknowns.

\section{RESULTS FOR A SECTOR BASIN}

To apply these numerical techniques to the ocean model, a single-hemispheric basin setup was chosen. The domain is a $60^{\circ}$-wide sector in longitude, with $\phi_{W}=290^{\circ}$ and $\phi_{E}=350^{\circ}$ 
between latitudes $\theta_{S}=10^{\circ} \mathrm{N}$ and $\theta_{N}=70^{\circ} \mathrm{N}$, which is comparable in size to the North Atlantic, and has a constant depth $D=4000 \mathrm{~m}$.

The surface buoyancy forcing is idealized, by prescribing the surface temperature $T_{S}$ and the surface freshwater flux $F_{S}$ as

$$
\begin{aligned}
T_{S}(\theta) & =\cos \left(\pi \frac{\theta-\theta_{S}}{\theta_{N}-\theta_{S}}\right) \\
F_{S}(\theta) & =\frac{\cos \left(\pi \frac{\theta-\theta_{S}}{\theta_{N}-\theta_{S}}\right)}{\cos \theta} .
\end{aligned}
$$

Note that in this case the dimensional meridional temperature difference over the sector is equal to $2 \eta_{T}$. The freshwater forcing is such that the integral over the surface is zero, which is a necessary condition for the existence of steady-state solutions. The wind forcing considered is an idealized profile for the North Atlantic representing a double gyre type wind stress [7], i.e., in dimensionless form

$$
\tau^{\phi}(\theta)=-\cos \left(2 \pi \frac{\theta-\theta_{S}}{\theta_{N}-\theta_{S}}\right) ; \quad \tau^{\theta}=0 .
$$

The dimensional temperature profile $T_{S}$, the freshwater flux $F_{S}$, and the wind stress pattern $\tau^{\phi}$ are shown in Fig. 2. Note that the freshwater flux becomes strongly negative in the northern region of the basin, because the size of the basin decreases.

Standard values of the dimensional and dimensionless parameters are listed in Table IV. Although the mixing of heat and salt is modeled in a crude way, by just assuming constant horizontal and vertical coefficients, the values listed in Table IV are typical for low-resolution ocean models. Below, we will also consider a case in which these values are increased to make computation over the different regimes easier. Note that in the standard case, there is no convective adjustment and the horizontal friction coefficient $A_{H}$ is rather large. The effect of convective adjustment on the solutions will be considered explicitly by varying the parameter $C_{a}$ or using the GAP, as explained above.

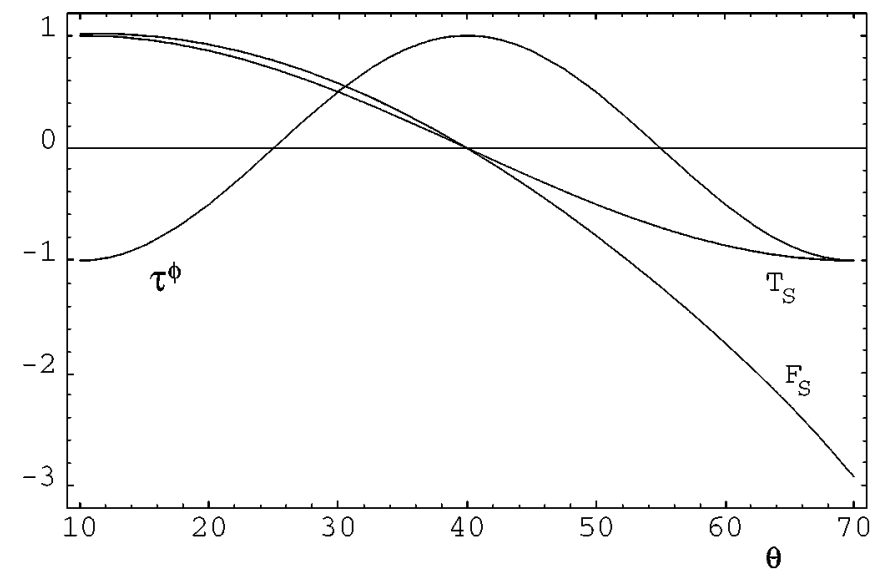

FIG. 2. Plot of the patterns of the forcing functions for the restoring temperature $T_{S}$, the freshwater flux $F_{S}$, and the zonal wind stress $\tau^{\phi}$. 
TABLE IV

Standard Values of Parameters Used in the

Numerical Calculations

\begin{tabular}{rlrl}
\hline $2 \Omega$ & $=1.4 \cdot 10^{-4}\left[\mathrm{~s}^{-1}\right]$ & $r_{0}$ & $=6.4 \cdot 10^{6}[\mathrm{~m}]$ \\
$\tau_{0}$ & $=1.0 \cdot 10^{-1}\left[\mathrm{~N} \mathrm{~m}^{-2}\right]$ & $D$ & $=4.0 \cdot 10^{3}[\mathrm{~m}]$ \\
$F_{0}$ & $=1.0 \cdot 10^{-7}\left[\mathrm{~m} \mathrm{~s}^{-1}\right]$ & $U$ & $=1.0 \cdot 10^{-1}\left[\mathrm{~m} \mathrm{~s}^{-1}\right]$ \\
$\rho_{0}$ & $=1.0 \cdot 10^{3}\left[\mathrm{~kg} \mathrm{~m}^{-3}\right]$ & $\Delta T$ & $=1.0[\mathrm{~K}]$ \\
$\alpha_{T}$ & $=1.6 \cdot 10^{-4}\left[\mathrm{~K}^{-1}\right]$ & $\tau_{T}$ & $=75[\mathrm{days}]$ \\
$A_{H}$ & $=1.6 \cdot 10^{7}\left[\mathrm{~m}^{2} \mathrm{~s}^{-1}\right]$ & $A_{V}$ & $=1.0 \cdot 10^{-3}\left[\mathrm{~m}^{2} \mathrm{~s}^{-1}\right]$ \\
$K_{H}$ & $=1.0 \cdot 10^{3}\left[\mathrm{~m}^{2} \mathrm{~s}^{-1}\right]$ & $K_{V}$ & $=1.0 \cdot 10^{-4}\left[\mathrm{~m}^{2} \mathrm{~s}^{-1}\right]$ \\
$\Delta S$ & $=1.0[0$ & $\alpha_{S}$ & $=7.6 \cdot 10^{-4}[-]$ \\
$C_{p}$ & $=4.2 \cdot 10^{3}\left[\mathrm{~J} \mathrm{~kg} \mathrm{~s}^{-1}\right]$ & $S_{0}$ & $=35.0[-]$ \\
$g$ & $=9.8\left[\mathrm{~m} \mathrm{~s}^{-2}\right]$ & $T_{0}$ & $=15.0[\mathrm{~K}]$ \\
$H_{m}$ & $=250\left[\mathrm{~m}^{c}\right]$ & $K_{V}^{c}$ & $=0.0\left[\mathrm{~m}^{2} \mathrm{~s}^{-1}\right]$ \\
\hline$R a$ & $=4.2 \cdot 10^{-2}$ & $\lambda$ & $=7.6$ \\
$\gamma$ & $=2.6 \cdot 10^{-2}$ & $P_{H}^{0}$ & $=1.5 \cdot 10^{-3}$ \\
$E_{H}$ & $=2.7 \cdot 10^{-3}$ & $P_{V}^{0}$ & $=3.9 \cdot 10^{-4}$ \\
$E_{V}$ & $=4.3 \cdot 10^{-7}$ & $B$ & $=10$ \\
$\eta_{T}$ & $=10.0$ & $\alpha_{\tau}$ & $=2.7 \cdot 10^{-2}$ \\
$C_{a}$ & $=0.0$ & $\epsilon_{R}$ & $=1.1 \cdot 10^{-4}$ \\
\hline
\end{tabular}

Note. Convective adjustment is turned off in the standard case.

The value of $A_{H}$ is bounded from below by the thickness of the boundary layers which develop near the continents. Near the western boundary, the Munk frictional boundary layer thickness at a latitude $\theta_{0}$ scales with $\left(A_{H} / \beta_{0}\right)^{1 / 3}$, where $\beta=2 \Omega_{0} \cos \theta_{0} / r_{0}$ monitors the variation of the Coriolis parameter. With a typical horizontal resolution of $3^{\circ}$, this leads to a typical lower bound of $A_{H}=2.5 \times 10^{4} \mathrm{~m}^{2} \mathrm{~s}^{-1}$ at $\theta_{0}=45^{\circ}$. However, the thickness of the Ekman layers near the continental walls have a typical width of $\left(A_{H} / f_{0}\right)^{1 / 2}$, where $f_{0}=2 \Omega_{0} \sin \theta_{0}$, which restricts the value of $A_{H}$ to be larger than $8 \times 10^{6} \mathrm{~m}^{2} \mathrm{~s}^{-1}$. To be on the safe side, we took a value twice the latter one. In typical ocean models, values much smaller are taken, but it has been shown that this leads to numerical waves near these boundaries which show up as wiggles in the steady-state solutions [23, 49]. It has furthermore been shown that this large value of $A_{H}$ does not affect the dominant geostrophic/hydrostatic balances over most of the domain. Consequently, for $A_{H}=1.6 \times 10^{7} \mathrm{~m}^{2} \mathrm{~s}^{-1}$, the "classical" $1 / 3$ power law is found in the relation between the overturning strength and the surface buoyancy forcing.

\subsection{Basic Bifurcation Diagrams}

In the first set of computations, steady states are computed as a function of the strength of the freshwater flux $\gamma$. By plotting a norm of the solution versus this control parameter, for every steady state computed, a so-called bifurcation diagram is obtained. As a norm, the dimensional maximum of the meridional overturning streamfunction $\left(\Psi_{M}\right)$ is chosen, which is computed as follows. The nondimensional overturning streamfunction $\Psi$ is defined by

$$
\bar{v}=\frac{\partial \Psi}{\partial z} ; \quad \bar{w}=-\frac{\partial \Psi}{\partial \theta},
$$


where

$$
\bar{v}=\int_{\phi_{W}}^{\phi_{E}} v \cos \theta d \phi ; \quad \bar{w}=\int_{\phi_{W}}^{\phi_{E}} w \cos \theta d \phi .
$$

With the scaling used, the maximum dimensional volume transport $\Psi_{M}=r_{0} U D \max |\Psi|$ and this is expressed in Sverdrups (Sv), where $1 \mathrm{~Sv}=10^{6} \mathrm{~m}^{3} \mathrm{~s}^{-1}$.

As a starting point, the two-dimensional case is considered, with zero wind forcing $\left(\alpha_{\tau}=0\right)$ and no rotation $\left(\eta_{f}=0\right)$. Zonally independent solutions can be found because of the free-slip boundary conditions on the east-west boundaries. For three different horizontal resolutions, $6^{\circ}, 3^{\circ}$, and $1.5^{\circ}$, each case with 16 equidistant vertical levels, the bifurcation diagrams are plotted in Fig. 3c. For this case, a value $K_{V}=10^{-3} \mathrm{~m}^{2} \mathrm{~s}^{-1}$ and $K_{H}=8 \times$

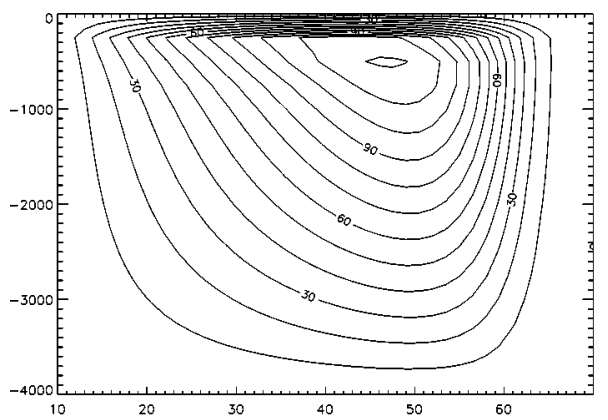

(a)

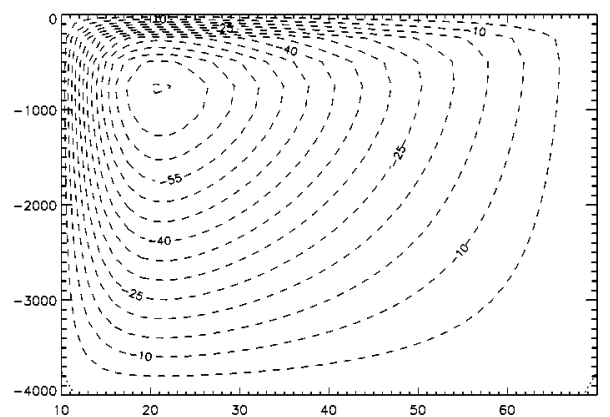

(b)

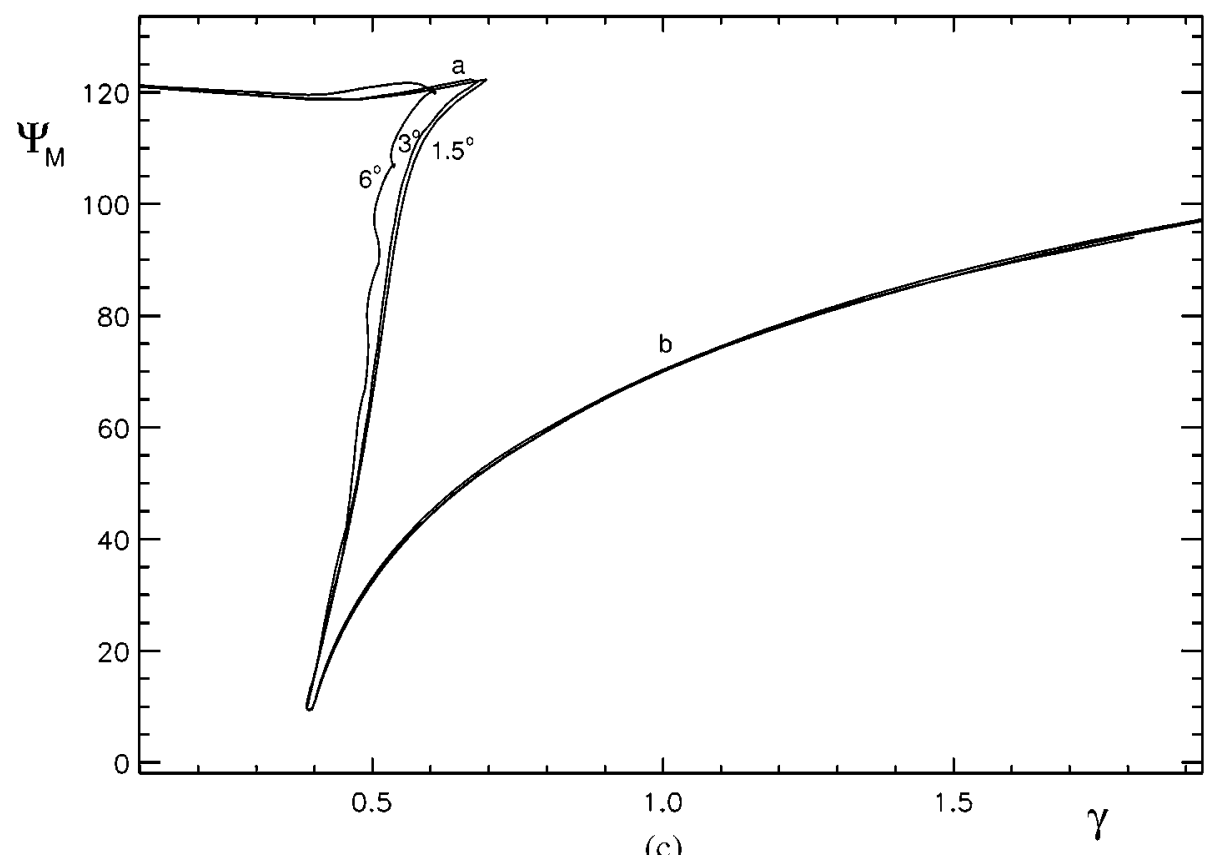

FIG. 3. (a)-(b) Meridional overturning streamfunctions $\Psi(\theta, z)$ for solutions at points marked (a) and (b) in panel (c) on the curve for $3^{\circ}$ resolution. (c) Bifurcation diagram for different horizontal resolutions and fixed 16 vertical levels. Maximum of the meridional overturning streamfunction $\left(\Psi_{M}\right)$ in Sv versus the strength of the freshwater forcing $(\gamma)$. 
$10^{6} \mathrm{~m}^{2} \mathrm{~s}^{-1}$ were taken. The structure of the bifurcation diagrams, with two saddle node bifurcations introducing a region of multiple equilibria is in correspondence with those from box-models $[39,42]$. The structure of the multiple equilibria is found for all the resolutions in Fig. 3c. However, the coarsest resolution results show spurious saddle node bifurcations due to numerical errors; these disappear on the finer grids.

A horizontal grid spacing of $3^{\circ}$ appears sufficient to capture the structure of the twodimensional flows. When $\gamma$ is small, the circulation is predominantly forced by the meridional temperature difference and the circulation is from equator to pole as in Fig. 3a, which shows the solution at point (a) in Fig. 3c. Because of the realistic temperature difference but the absence of rotation, the overturning is much too large compared to reality. The first saddle node bifurcation occurs at $\gamma=0.7$ and an unstable branch exists down to the second saddle node at $\gamma=0.4$. Along this branch, the solution changes from temperature controlled, with overturning in the north, to salt controlled with overturning in the south. On the stable branch for larger $\gamma$, the surface flow is from pole to equator and hence predominantly forced by the meridional salinity gradient (Fig. 3b).

The bifurcation diagram obtained with $3^{\circ}$ horizontal resolution in Fig. 3 is replotted in Fig. 4a as the dotted curve. The "deformation" of this bifurcation diagram when rotation is added (by increasing $\eta_{f}$ from zero to one) and wind forcing (increasing the value of $\alpha_{\tau}$ from 0 up to its standard value) shows that with rotation, the multiple equilibria structure disappears (dashed curve in Fig. 4a). The addition of wind does not change the bifurcation diagram (drawn curve in Fig. 4a) qualitatively, although the strength of the overturning changes.

In Fig. 4b, the bifurcation diagram for standard values of parameters as in Table IV is plotted, which again shows the typical multiple equilibria structure also found in the high thermal diffusion two-dimensional case (Fig. 3a, dotted curve). Note that by decreasing the thermal diffusivity $K_{V}$, the overturning has decreased substantially, because of a reduction of the overall meridional buoyancy gradient. Consequently, the range of $\gamma$ where the multiple equilibria occur is shifted to much smaller values. The results indicate that there is a qualitative correspondence between three-dimensional solutions and twodimensional solutions (and eventually box models) with respect to the existence of multiple equilibria [28]. However, the regimes of existence in parameter space may substantially differ.

To show the main characteristics of the three-dimensional flows, in addition to the overturning streamfunction, also the velocity field at 250-m depth and meridional sections of the density and velocity field near the eastern boundary (where the largest gradients in the solutions appear) at $\phi=347^{\circ}$ are plotted. The solution at point (a) in Fig. 4a has an overturning of about $24 \mathrm{~Sv}$ (Fig. 5a). The main sinking area is located near $50^{\circ} \mathrm{N}$ (Figs. 5b-5d) and the flow has a strong zonal component at all latitudes. In the upper layer flow, one can see the effect of the Ekman mass transport, which is always perpendicular and to the right of the wind. For example, at $40^{\circ} \mathrm{N}$ the wind is directed eastward, which gives a southward Ekman transport. In the low latitude area, the thermally driven overturning and the Ekman transport are in the same direction and hence the northward flow is stronger. Because there is no convective adjustment, the flow is not stably stratified (Fig. 5c), which causes the southerly position of the sinking region (Fig. 5d). Upwelling occurs mainly near the southern and western boundary (Fig. 5b) and the downwelling is concentrated in a relatively small area near the eastern boundary and in the central part of the basin. 


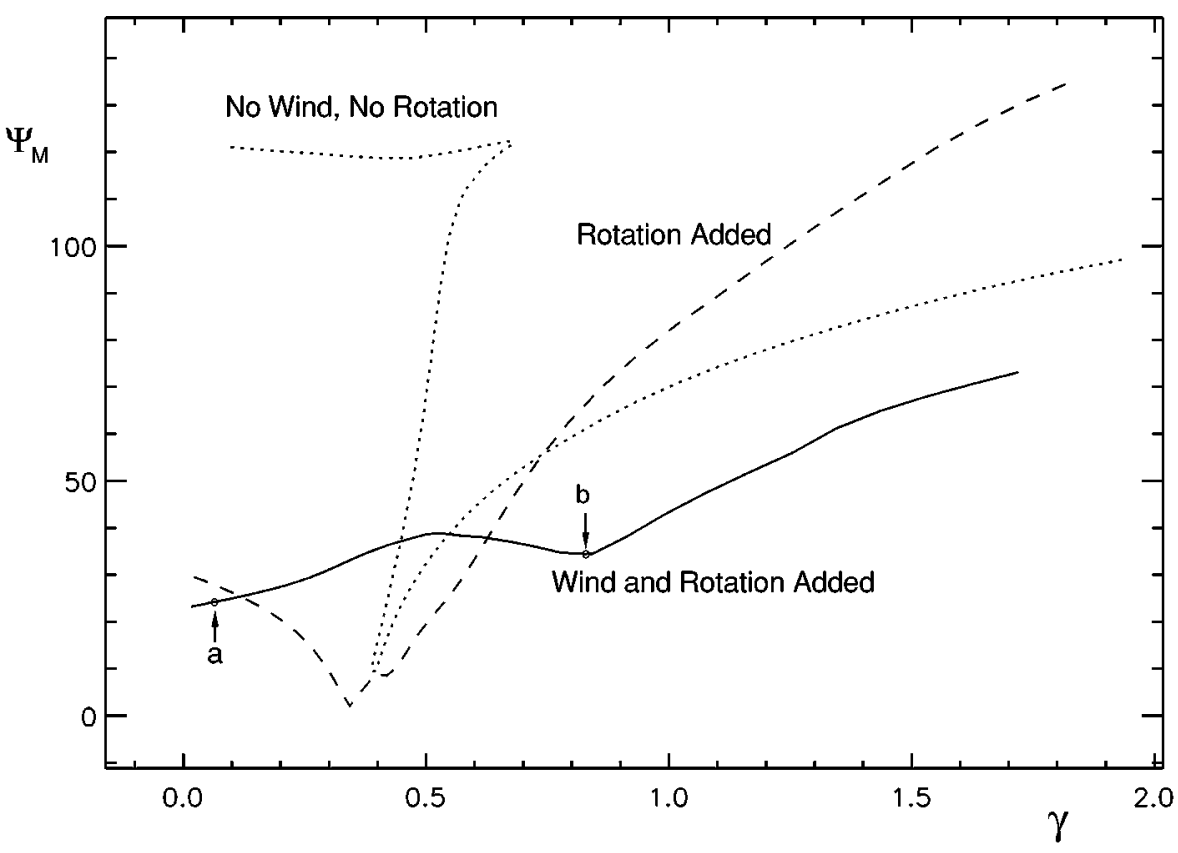

(a)

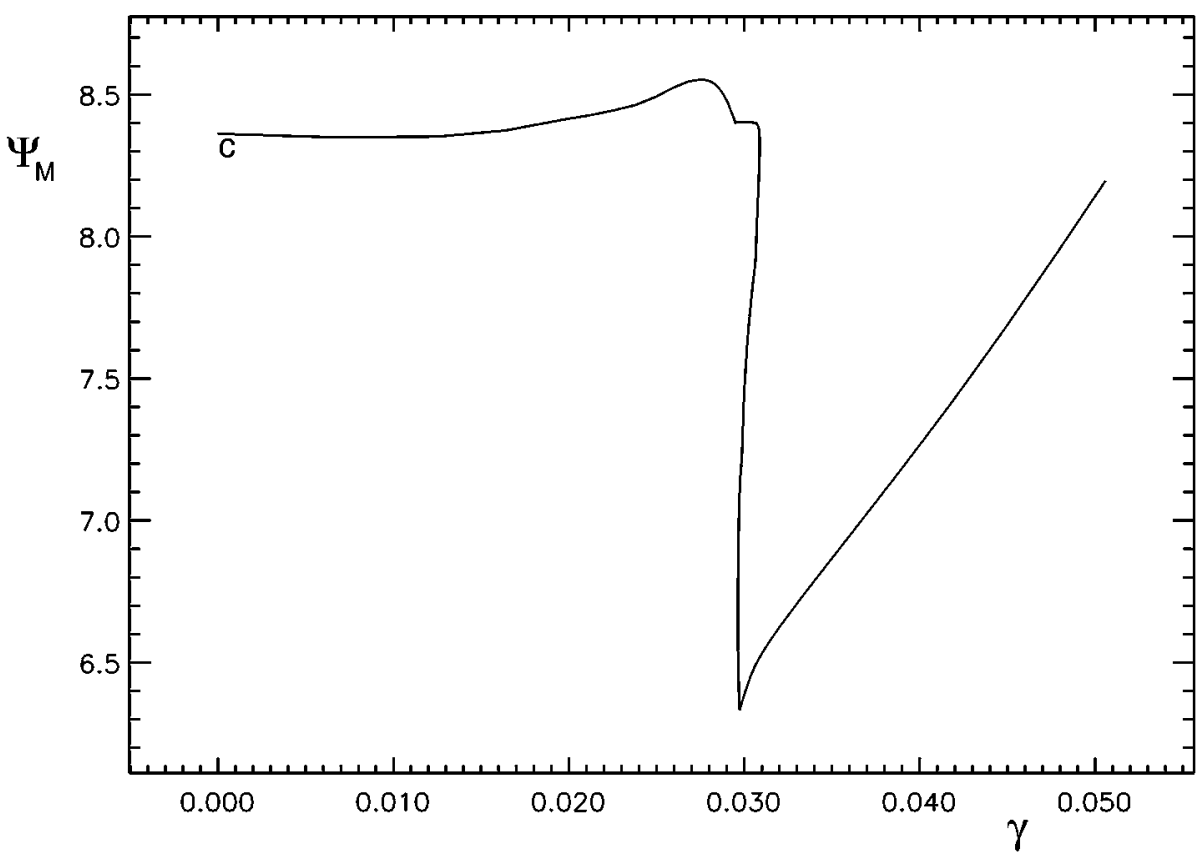

(b)

FIG. 4. (a) 'Deformation' of the bifurcation diagram for the two-dimensional high diffusion case $\left(K_{H}=\right.$ $8 \times 10^{3} \mathrm{~m}^{2} \mathrm{~s}^{-1}$ and $K_{V}=10^{-3} \mathrm{~m}^{2} \mathrm{~s}^{-1}$ ) for $\eta_{f}=0$ and no wind (dotted curve) to the bifurcation diagram for $\eta_{f}=1$ and full wind (drawn curve). An intermediate result, where wind forcing is absent (with $\eta_{f}=1$ ) is also shown (dashed curve). For all curves, the maximum of the meridional overturning streamfunction $\left(\Psi_{M}\right)$ is plotted versus the strength of the freshwater forcing $(\gamma)$. (b) Bifurcation diagram for standard values of parameters as in Table IV for the case where both wind and rotation are included. 


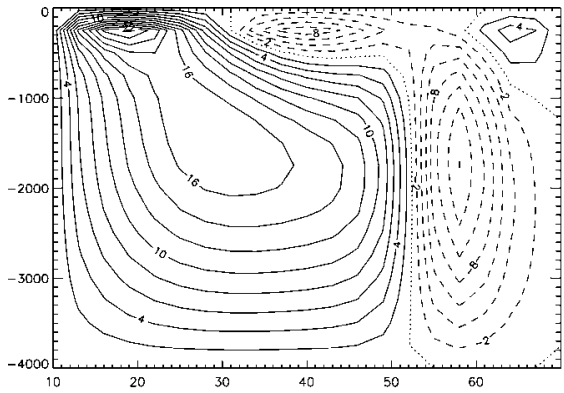

(a)

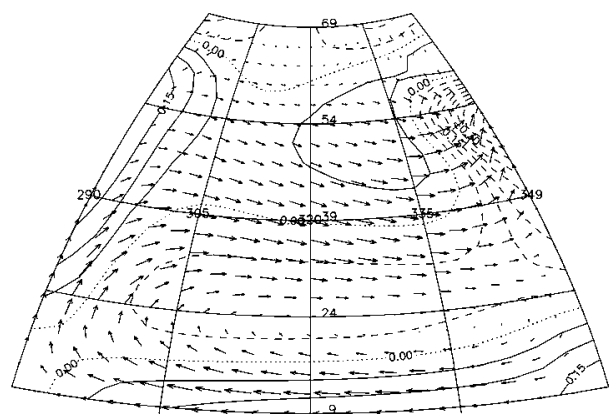

(b)

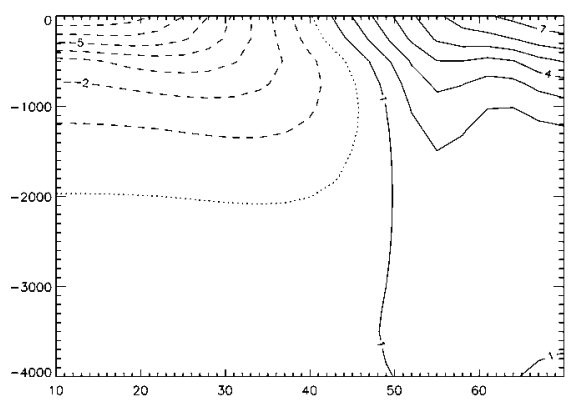

(c)

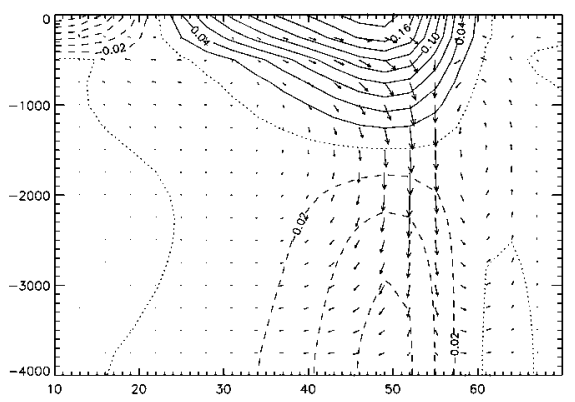

(d)

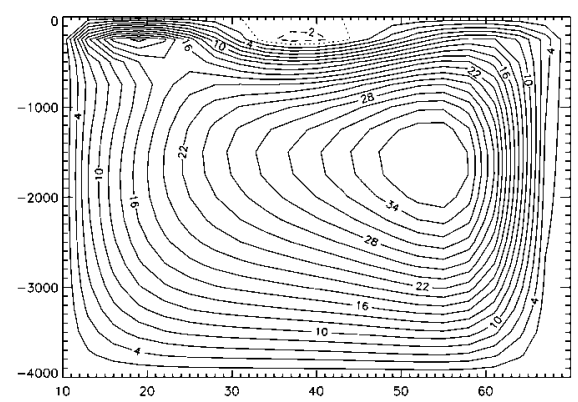

(e)

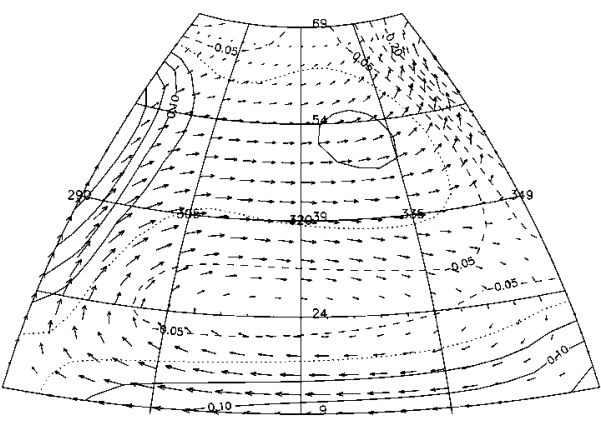

(f)

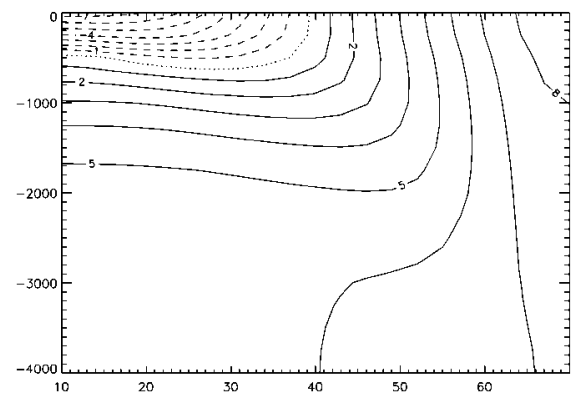

(g)

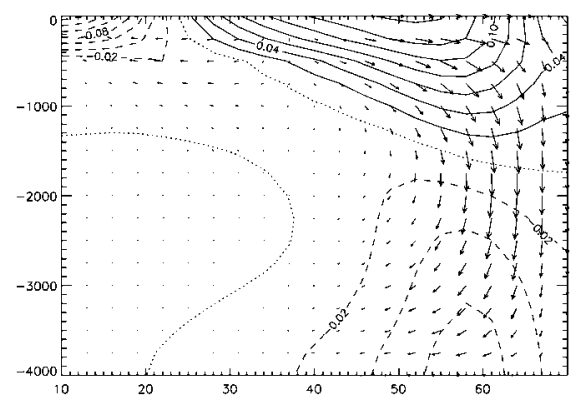

(h)

FIG. 5. (a)-(d) Flow pattern of the steady solution at the point labeled (a) in Fig. 4a. (e)-(f) Flow pattern of the steady solution obtained from (a)-(d) with $C_{a}=250$. (a) and (e) Meridional overturning stream function (in Sverdrups). (b) and (f) Velocity field near the surface (at $250 \mathrm{~m}$ depth). In this plot, vectors indicate the horizontal velocity $(u, v)$ at this depth and the contours represent the dimensionless vertical velocity, $w$. Solid lines represent upwelling (flow out of the plane), dashed lines downwelling (flow into the plane). (c) and (g) Density (dimensionless) and (d) and (h) velocity plot for a north-south vertical plane at a grid point just west from the eastern boundary $\left(\phi=347^{\circ}\right)$. In the latter picture, the vectors indicate the $(v, w)$ velocity field, whereas the contours represent the zonal velocity, $u$ (again dimensionless). 
For comparison, with $C_{a}=250$ (and for other parameter having the same values), the solution at point (a) in Fig. 4a transforms into that shown in Figs. 5e-5h. The enhanced mixing causes the overturning to extend to the northern boundary (Fig. 5e) and the strength of the overturning increases from about $24 \mathrm{~Sv}$ to $39 \mathrm{~Sv}$. The surface velocity field is only slightly changed and mainly the position of the sinking region has shifted northward (Figs. $5 \mathrm{f}-5 \mathrm{~h}$ ). The stratification is statically stable, except in the far north (Fig. $5 \mathrm{~g}$ ).

In the standard case (Fig. 4b), the solutions for the location labeled (c) are shown in Fig. 6. For the standard case $C_{a}=0$, the overturning flow (about $8 \mathrm{~Sv}$ ) now extends to the northern boundary (Figs. 6a and 6b). The effect of a smaller value of $K_{V}$ is clearly seen in the vertical structure of the density field which is much more confined to the upper layers (Fig. 6c). The latter holds also for the flow field and consequently the sinking area is more confined to the north (Fig. 6d) than for the higher $K_{V}$ flows in Fig. 5. For the same value of the parameters, the completely statically stable solution, computed with the GAP, has an overturning of $12 \mathrm{~Sv}$ (Fig. 6e). The flow field and density field are fairly similar to the statically unstable solution, except in the northern regions (Figs. 6f-6h).

In each of the cases in Fig. 4, the flow becomes salinity controlled at larger $\gamma$, which leads to a southern sinking solution. For example, at the location labeled (b) in Fig. 4a, the sinking region of this solution (not shown) is located near $20^{\circ} \mathrm{N}$, whereas the overturning is about $30 \mathrm{~Sv}$. Most of the downwelling occurs in the southern and western part of the basin.

\section{STABILITY OF STEADY STATES}

In the previous section, it was shown that branches of steady states could be computed as a function of the freshwater flux strength $\gamma$ using continuation methods. In this section, the stability of the solutions on these branches is addressed by solving the linear stability problem with the Jacobi-Davidson QZ method. Part of the (drawn) branch of solutions in Fig. 4a is replotted in Fig. 7a, where the location of point (a) is again labeled. The stability of the solutions is now indicated by the line style: a solid line style indicates stability while a dotted linestyle denotes an unstable branch. Bifurcations are indicated by markers and a triangle indicates a Hopf bifurcation.

The real and imaginary part of an eigenvector $\mathbf{x}=\mathbf{x}_{R} \pm i \mathbf{x}_{I}$ corresponding to a complex conjugate pair of eigenvalues $\sigma=\sigma_{r} \pm i \sigma_{i}$ provide the time periodic disturbance structure $P(t)$ with angular frequency $\sigma_{i}$ and growth rate $\sigma_{r}$ which oscillates around the steady state, i.e.,

$$
P(t)=e^{\sigma_{r} t}\left[\mathbf{x}_{R} \cos \left(\sigma_{i} t\right)-\mathbf{x}_{I} \sin \left(\sigma_{i} t\right)\right] .
$$

The evolution of this perturbation can be followed by looking for example at $P\left(\frac{-\pi}{2 \sigma_{i}}\right)=\mathbf{x}_{I}$ and then at $P(0)=\mathbf{x}_{R}$.

For the high diffusion case, the real part (marked with a diamond) and imaginary part (marked with a square) of the "most dangerous" modes are plotted in Fig. 7b, with a corresponding line style indicating the same eigenpair. At $\gamma=1.55 \times 10^{-2}$, the left endpoint of the curve in Fig. 7a, the first mode (dotted curve in Fig. 7b) is stationary with slightly negative real part. The next "most dangerous" mode is an oscillatory mode (drawn curves in Fig. 7b) having a frequency of $\sigma_{i}=0.027$, which corresponds to a period $\mathcal{P}=2 \pi r_{0} /\left(U \sigma_{i}\right) \approx$ 400 years. When $\gamma$ increases, the stability of these modes is not affected much, but another oscillatory pair shows up. This mode destabilizes near $\gamma=5.07 \times 10^{-2}$, the latter 


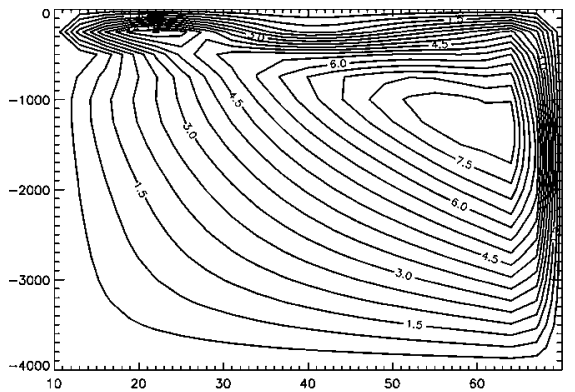

(a)

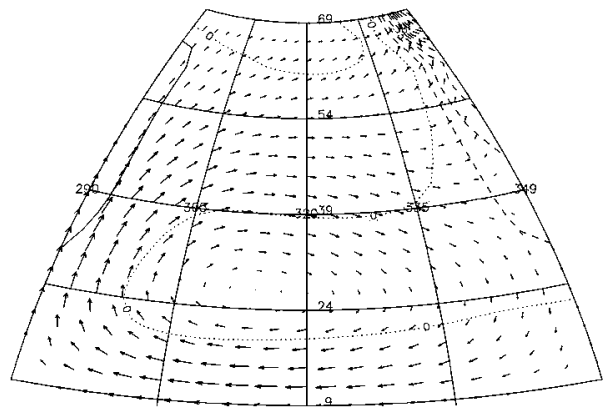

(b)

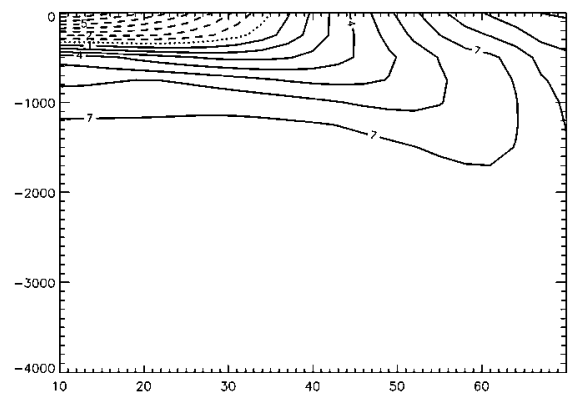

(c)

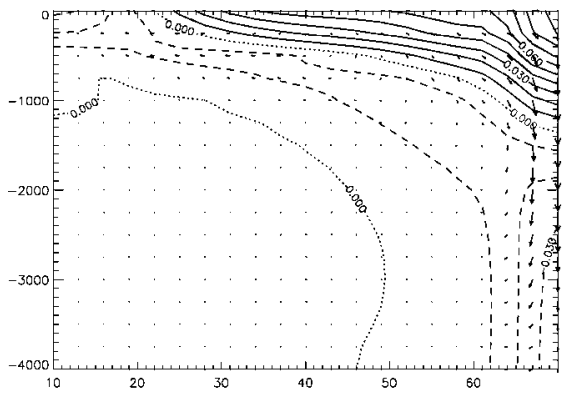

(d)

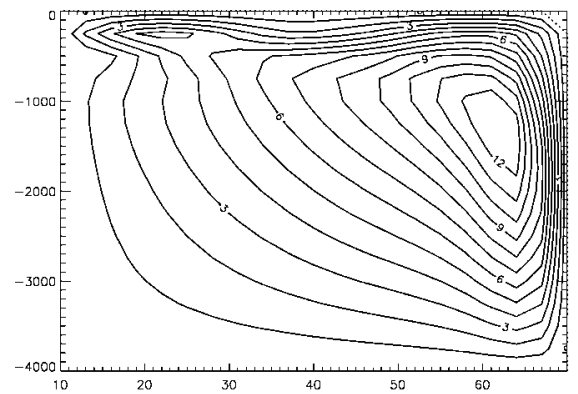

(e)

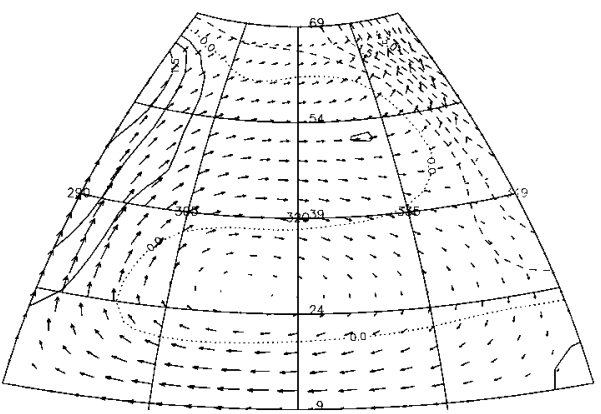

(f)

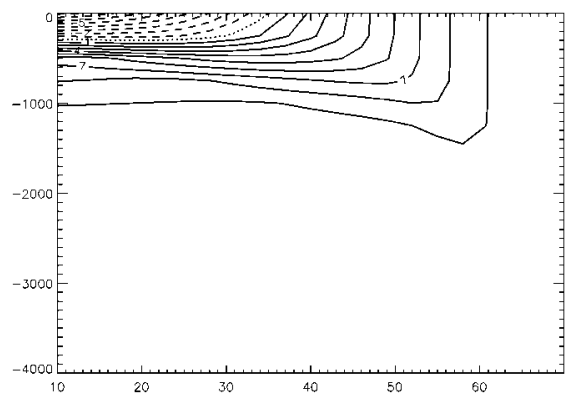

(g)

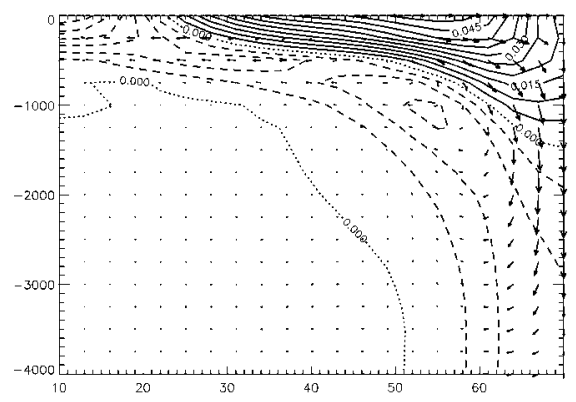

(h)

FIG. 6. (a)-(d) Flow pattern of the steady solution at the point labeled (c) in Fig. 4b. (e)-(f) Flow pattern of the steady solution obtained from (a)-(d) with the GAP. (a) and (e) Meridional overturning stream function (in Sverdrups). (b) and (f) Velocity field near the surface (at $250 \mathrm{~m}$ depth). In this plot, vectors indicate the horizontal velocity, $(u, v)$ at this depth and the contours represent the dimensionless vertical velocity, $w$. Solid lines represent upwelling (flow out of the plane), dashed lines downwelling (flow into of the plane). (c) and (g) Density (dimensionless) and (d) and (h) velocity plot for a north-south vertical plane at a grid point just west from the eastern boundary $\left(\phi=347^{\circ}\right)$. In the latter picture, the vectors indicate the $(v, w)$ velocity field, whereas the contours represent the zonal velocity, $u$ (again dimensionless). 


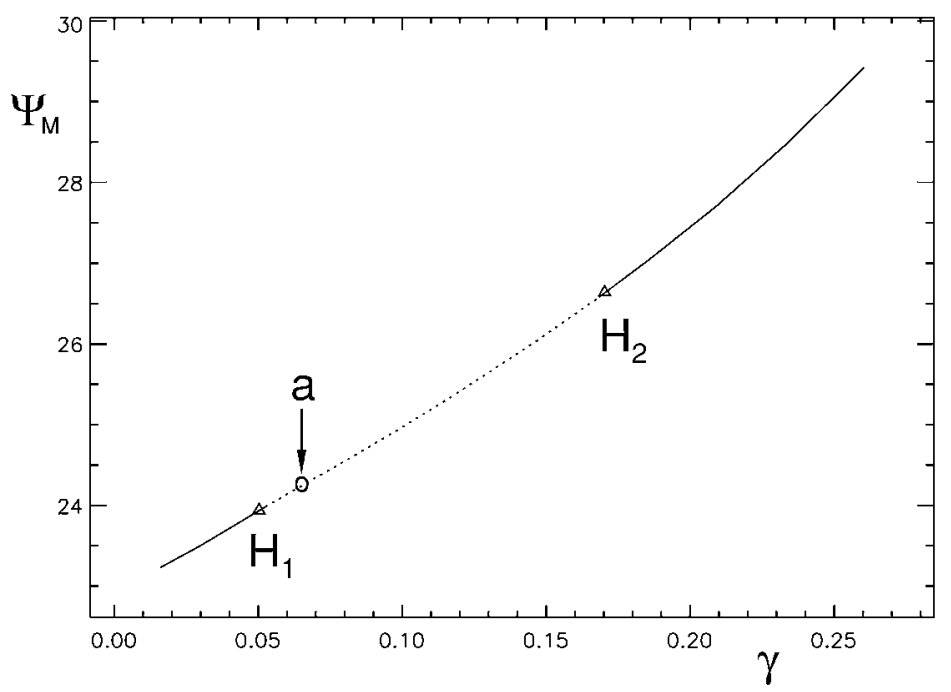

(a)

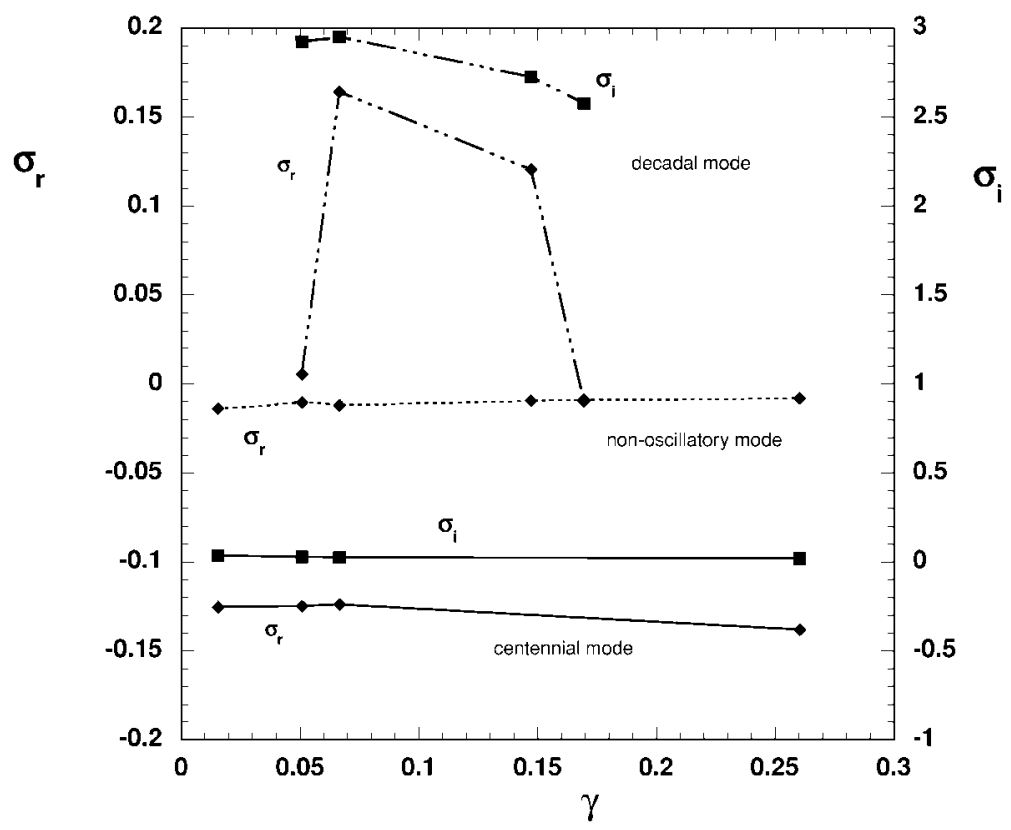

(b)

FIG. 7. (a) Bifurcation diagram for the case $\eta_{f}=1.0$ and $C_{a}=0$, similar to the drawn curve in Fig. 4a. The stability of the steady solutions is now indicated by its line style: a solid line style indicates stability while a dotted linestyle denotes an unstable branch. Bifurcations are indicated by markers, a triangle indicating a Hopf bifurcation. (b) Real $\left(\sigma_{r}\right)$ and imaginary $\left(\sigma_{i}\right)$ part of the "most dangerous" eigenvalues along the branch in (a). Similar linestyle indicates the same eigenpair and real and imaginary parts are labeled.

corresponding to the location of $H_{1}$ in Fig. 7a. The time scale of oscillation of this mode $\left(\sigma_{i}=2.92\right)$ is about 4 years. In the high diffusion case, the decadal mode turns out to be sensitive to the changes in the stratification in the northern basin arising through the application of convective adjustment is applied. However, the centennial modes and the nonoscillatory mode are very robust. 


\section{TABLE V}

Eigenvalues $\sigma=\sigma_{r} \pm i \sigma_{i}$ of the Most Dangerous Eigenmodes for (1) the Solution in Figs. 6a-6d under Restoring Conditions and (2) for the Solution in Figs. 6e-6h under Prescribed Flux Conditions

\begin{tabular}{ccccc}
\hline Eigenvalue & $\sigma_{r}^{1}$ & $\sigma_{i}^{1}$ & $\sigma_{r}^{2}$ & $\sigma_{i}^{2}$ \\
\hline 1 & $-3.5 \times 10^{-3}$ & 0.0 & 0.0 & 0.0 \\
2 & $-2.3 \times 10^{-2}$ & 0.0 & $-1.6 \times 10^{-2}$ & 0.0 \\
3 & $-2.5 \times 10^{-2}$ & 0.0 & $-2.5 \times 10^{-2}$ & 0.0 \\
$4-5$ & $-2.6 \times 10^{-2}$ & $\pm 1.4 \times 10^{-2}$ & $-2.8 \times 10^{-2}$ & $\pm 1.7 \times 10^{-2}$ \\
\hline
\end{tabular}

Note. An entry ' 0.0 ' indicates a value smaller than $10^{-5}$.

In the standard case, the first six eigenvalues determining the stability of the solution at location (c) in Fig. 4b are shown in Table V. The first three of these modes are nonoscillatory modes, but the next two form a complex conjugate pair with centennial oscillation period. The overturning streamfunction and the velocity field at mid-depth of this oscillatory mode are plotted in Figs. 8a-8d. These patterns are very similar to the overturning oscillation found in two-dimensional models [12], which are caused by the propagation of salinity anomalies along the mean overturning flow. The oscillation can be seen as a periodic weakening and strengthening of the basic state overturning.

In Table V, also the leading eigenvalues are shown for the most "realistic" case within this idealized model and geometry. The stability is computed of the completely statically stable solution shown in Figs. 6e-6h under prescribed flux conditions for the temperature. For the latter, the usual procedure is to diagnose the heat flux from the steady state and compute the stability under this diagnosed flux [18]. Because the perturbation temperature is determined up to an additive constant, now an eigenvalue zero must appear (confirmed numerically in Table V). The next two eigenvalues are real and eigenvalues 4 and 5 form a complex conjugate pair, having approximately the same oscillation frequency and growth rate as the centennial mode in Figs. 8a-6d. Patterns of the overturning and mid-depth velocity of this oscillatory mode are also plotted in Figs. 8e-8h and show indeed a close correspondence with those in Figs. 8a-8d.

\section{TRANSIENT THERMOHALINE FLOWS}

In this section, examples of transient flows, computed with the implicit time-stepping method, are shown. Having the information of the steady states and their stability immediately provides guidelines for the interesting areas in parameter space. The latter is the region between $H_{1}$ and $H_{2}$ in Fig. 7a, where limit cycles are expected and that arise through supercritical Hopf bifurcations.

However, first the transient behavior due to parameter variation will be considered. As an example, point (a) in the bifurcation diagram in Fig. 4a is taken as initial condition and at $t=0$, the parameter $C_{a}$ is increased from 0 to 250 . The development of the flow with time toward the steady solution in Figs. $5 \mathrm{e}-5 \mathrm{~h}$ is monitored and the maximum overturning $\Psi_{M}$ is plotted in Fig. 9a. Each time step is indicated with a marker and the dimensional values are given in years. Initially, relatively small time steps have to be taken, because the solution 


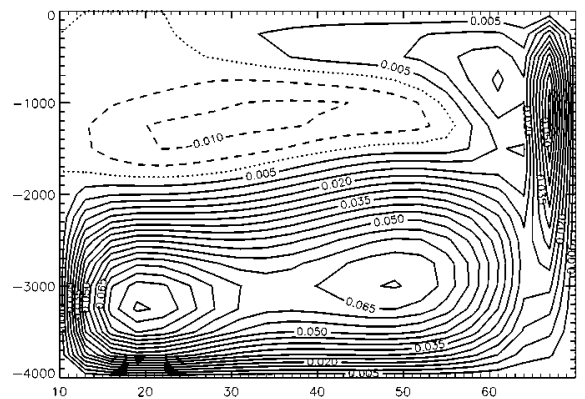

(a)

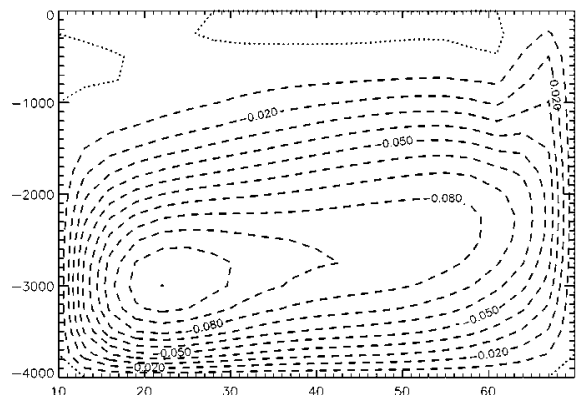

(b)

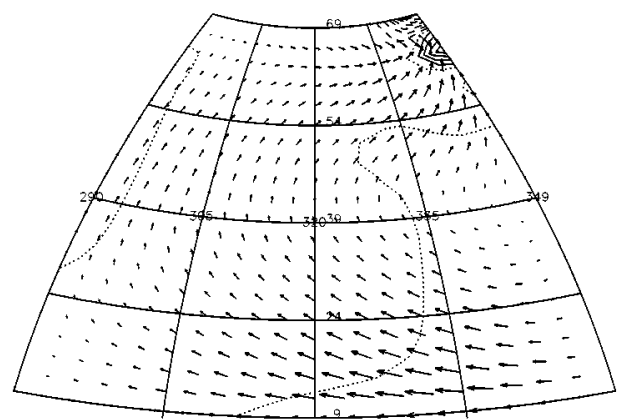

(c)

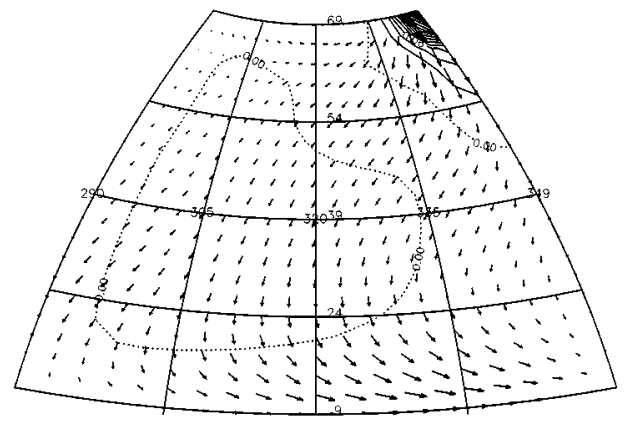

(d)

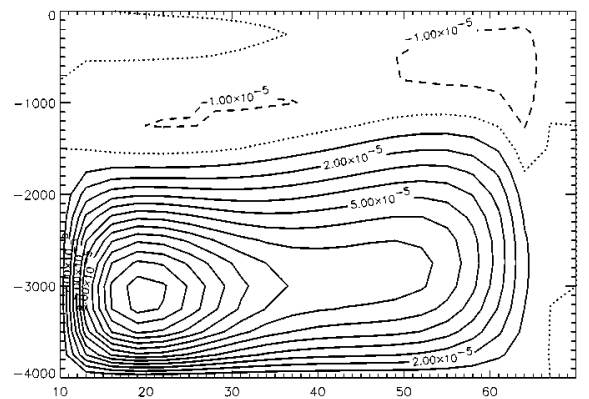

(e)

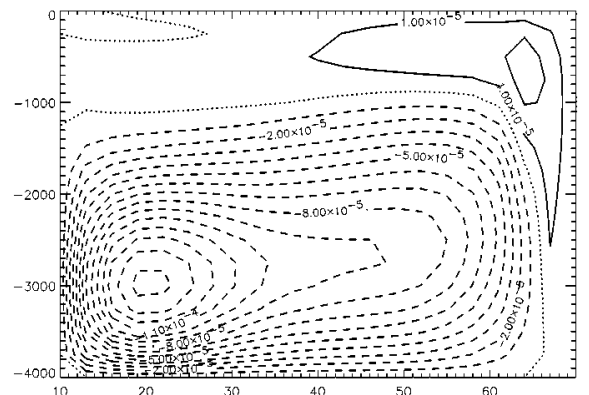

(f)

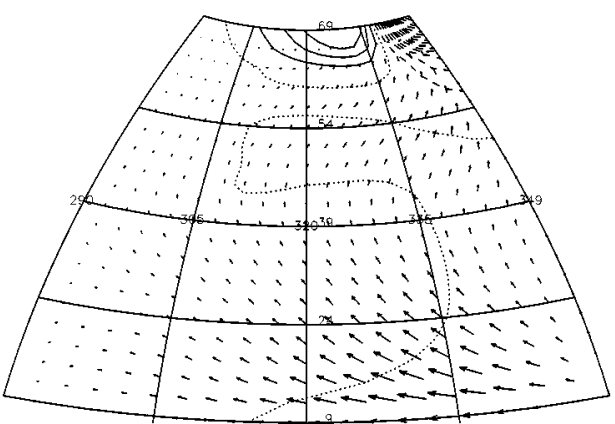

(g)

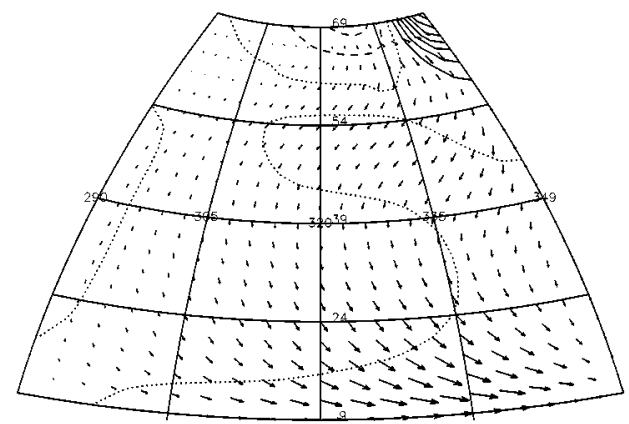

(h)

FIG. 8. (a)-(d) Oscillatory eigenmode pair 4-5 for the solution in Fig. 6a-6d under restoring conditions. (e)-(h) Same eigenpair but now for the statically stable solution in Fig. 6e-6h under prescribed flux conditions. (a) and (e) Real part of the meridional overturning stream function. (b) and (f) Imaginary part of the meridional overturning stream function. (c) and (g) Real part of the velocity field at $2000 \mathrm{~m}$ depth. (d) and (h) Imaginary part of the velocity field at $2000 \mathrm{~m}$ depth. 


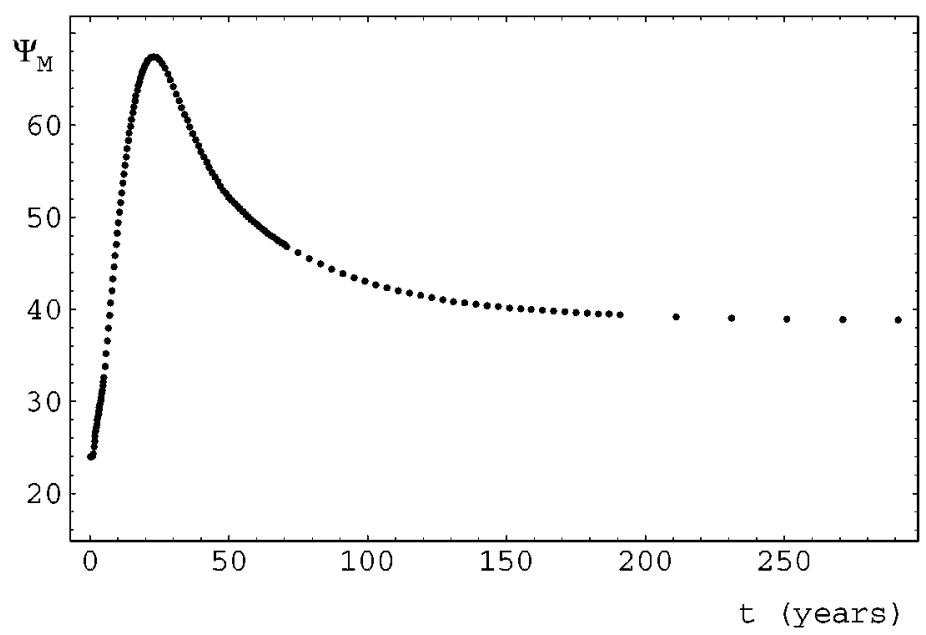

(a)

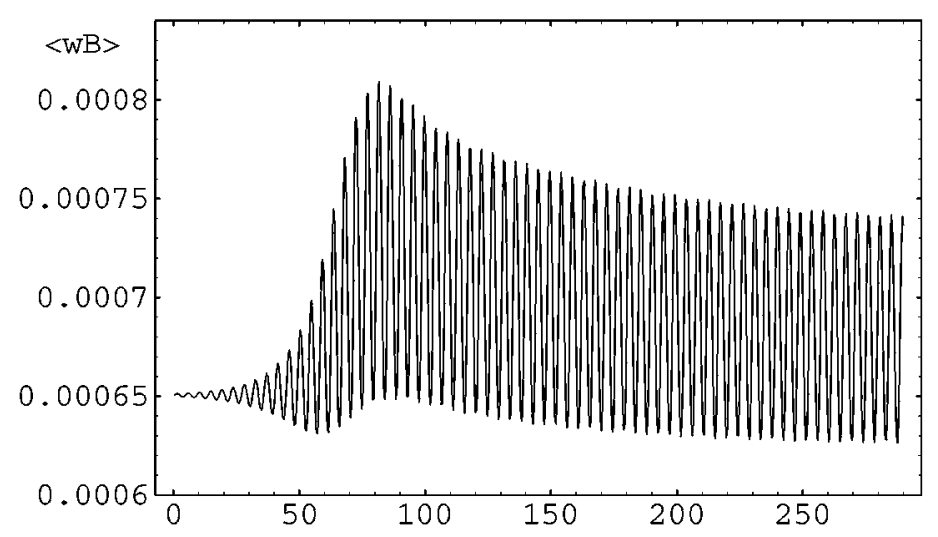

(b)

FIG. 9. (a) Overturning strength in Sverdrups versus dimensional time in years. The starting point is point (a) in Fig. 4a and initially, the parameter $C_{a}$ is set at the value 250. Each point indicated gives the actual time step taken. (b) Plot of the dimensionless buoyancy production $\langle w B\rangle$ (Volume integral of vertical velocity, $w$, times buoyancy, $B=R a(T-\lambda S)$ ) versus time. The trajectory started at the same point as in (a), where the steady state is unstable for $C_{a}=0$. Here, a limit cycle is reached after an initial growth time of the instability of about 100 years.

changes quite a bit in the northern region. However, during the approach to equilibrium, time steps of up to 50 years can be taken. This clearly demonstrates the big advantages of implicit techniques when investigating sensitivity of solutions to parameter changes.

Results of total time to compute a solution for a certain time step $\Delta t$ are presented in Table VI, where the initial condition is the last computed point in Fig. 9a. Here again, the scaling 1 in Table I and the value of the drop-tolerance $\varepsilon=1.2 \times 10^{-3}$ was used. Increasing the time step by a factor 100 increases the total time only be a factor 4 , which is mainly due to the increased number of Newton iterations needed. The fill and also the number of iterations in BICGSTAB are not much affected by the magnitude of the time step.

As a second example, we investigate the finite amplitude decadal oscillation by starting with a steady solution at point (a) in Fig. 7a and perturb it slightly. After an initial growth time of the instability, which is about 100 years, a periodic orbit is reached and it can be 
TABLE VI

The effect of the Time Step on the Performance of the Newton Process and Iterative Solver During the Implicit Time Integration

\begin{tabular}{lrrrrr}
\hline$\Delta t$ (year) & 0.5 & 1.0 & 4.0 & 20.0 & 50.0 \\
\hline Time (sec) & 50 & 53 & 73 & 98 & 198 \\
Max. nonzero & 129 & 133 & 138 & 143 & 144 \\
Max. iteration & 16 & 16 & 16 & 22 & 29 \\
Newton It. & 3 & 3 & 4 & 5 & 10 \\
\hline
\end{tabular}

followed with a time step of 0.5 year. The period is about 4 years, which is in correspondence with the period determined from the eigenmode at Hopf bifurcation. Here, the combination of continuation techniques, eigenvalue solvers, and implicit time integration clarifies the origin of the oscillation as an instability of the steady flow.

The time steps, such that sufficiently accurate solutions are obtained during the implicit time integration, can be determined by comparing the results of computations over a certain time interval with several (in most cases three) different time steps. With the CrankNicholson scheme being second-order accurate, in this way also the absolute accuracy can be determined. For the case in Fig. 9a, different time steps are therefore taken in the beginning of the integration than those in later stages. For the case in Fig. 9b, a fixed time step was taken since the solution oscillates with fixed frequency.

\section{DISCUSSION}

In this paper, we have presented a new fully implicit model of the thermohaline ocean circulation suitable for the study of long time scale variability, such as centennial and larger time scale oscillations. The results shown for a sector model are the first of its kind, where fully implicit techniques are used and where indeed very long time step can be taken. This makes the approach very well suited for sensitivity studies, similar to those which have been done for the two-dimensional flows [12, 44].

The key to being able to use these long time steps is the solution of the large linear systems of equations with iterative solvers. The combination of the MRILU preconditioning technique with the BICGSTAB solver enables one to compute solutions to the steady equations. Tuning of the parameters in MRILU is required and rescaling of the Jacobian matrix is necessary to achieve efficiency. Once this has been done for the steady case, the performance of MRILU improves in the time-dependent implicit time stepping case, since the Jacobian matrix becomes better conditioned. All computations in the paper, which are still for a low resolution ocean model set-up ( $3^{\circ}$ horizontally and 16 levels vertically), could therefore be performed on a XP1000 $500 \mathrm{MHz}$ workstation with 1 GB internal memory. The use of MRILU is not restricted to this particular application, since it has a large amount of flexibility. It can be used on both structured and unstructured grids, with equidistant and nonequidistant grid spacing and there is a recipe available to tune the parameters within the factorization step.

The combination of a continuation method for computing steady states, the JacobiDavidson QZ method for the linear stability problem, and implicit time-stepping techniques for monitoring the time-dependent flow provides a powerful tool to understand the structure 
of the thermohaline flow solutions in parameter space. For the simple single basin configuration, the results presented here show that one is able to trace steady solution branches into a relevant parameter regime and to determine the most dangerous eigenmodes. It shows that multiple equilibria in the high-diffusion, two-dimensional case disappear when rotation is taken into account, but that these reappear in the rotational standard (low diffusion) regime.

In addition, decadal oscillations appear as instabilities on the three-dimensional wind and thermohaline driven flow. For the high diffusion case considered here, the unstable stratification in the northern region of the basin turns out to be important, since these modes stabilize when the steady flow is statically stable. However, in the standard case, these type of modes arise as interdecadal instabilities [40] when the value of $K_{H}$ is decreased. These modes can indeed be related to the many examples of (inter)decadal variability found in low resolution ocean models [21]. The results also indicate that centennial oscillations are a very robust feature within these models, but they turn out to be stable under the forcing and parameters chosen here. It appears that these modes become exited in a slightly different parameter regime (smaller $K_{H}$ ) when stochastic noise is included in the heat flux forcing to give centennial oscillatory behavior, superposed on the dominant interdecadal behavior.

The ocean model used here is one which contains the basic fluid dynamics, but it is still some distance from "state of the art" low-resolution ocean models. However, there is no principle difficulty to bridge this gap and in effect, many of the intermediate steps have already been taken. The only technical difficulty comes from the computation of the Jacobian matrix and the ability to solve the linear systems of equations. Continental geometry and bottom topography can be easily included, by substituting equations (representing boundary conditions) at matrix level, similar to that done in shallow water models [35]. There is also no principle difficulty in implementing a nonlinear equation of state, although a dependency of the density on pressure complicates matters technically. The ocean model has already been coupled to an energy balance atmosphere model, and changes in surface boundary conditions are easily implemented. As in many ocean models, however, the issue of representing the nonresolved scales (mixing) is difficult. There is no principle difficulty in including a rotation of the mixing tensor to represent the dominant mixing along isopycnal surfaces and to reduce diapycnal mixing. Also, a full parameterization as suggested in [15] can be included, although technical difficulties in calculating the Jacobian have to be overcome. Periodic boundary conditions can also be handled without any trouble, because the MRILU method, used to solve the linear systems, does not require any preferred sparsity (i.e., banded) structure of the Jacobian matrix.

The results presented here are for a model configuration, for which the value of the horizontal friction is orders of magnitude larger than that considered realistic for the ocean and two orders of magnitude larger than those used in low-resolution ocean models. This is necessary because of problems in resolving the horizontal (steady) Ekman boundary layers, which contain large velocity gradients. In explicit models, the time step is small enough to allow adjustment of numerical errors due to nonresolved boundary layers through Kelvintype waves. The consequence is that no wiggles appear, but the solutions will effectively never reach steady state. The issue how to handle these nonresolved boundary layers in a steady state or large time step set-up is outside the scope of this paper. We want to stress that high viscosity is not a restriction of the use of the methodology of continuation and implicit time stepping, which has been the focus of this paper. When the horizontal viscosity is taken spatially dependent and artificially increased only near the southern and eastern boundaries, flows can be computed for much smaller interior horizontal viscosities. 
Even with the present restriction of these fully implicit models to high viscosity, many interesting and physically relevant problems still can be tackled. Indeed, for high viscosity, the flows computed satisfy geostrophic and hydrostatic balances, but horizontal friction enters at leading order in the interior potential vorticity balance. Under wind forcing only, the interior flow has a small east-west asymmetry, with little developed western boundary current. When the horizontal friction is decreased, the east-west asymmetry and the strength of the gyres increases. However, down to horizontal viscosity values used in low-resolution explicit ocean models, there will be a unique wind-driven solution and the nonlinear western boundary current regime is not yet reached. Although potentially this change of flow may have a strong impact on the steady states of the full three-dimensional flows in these sector models, we think that there will be no changes in the qualitative structure of the bifurcation diagrams.

The analysis of the physical mechanisms of the existence of both the multiple equilibria and the oscillatory modes of variability $[40,47]$ indicates that these phenomena are thermodynamically controlled. For example, the salt-advection feedback is responsible [11] for the occurrence of the multiple equilibria, while for the (inter)decadal modes of variability, eastwest propagation of density anomalies and their effect on the meridional and zonal overturning are essential [40]. In these cases, the details of the momentum balances are not crucial as long as a flow response is generated to density anomalies. For essentially three-dimensional features, such as the (inter)decadal oscillations, a geostrophic/hydrostatic response is required, which can be represented in the high viscosity models used here [40]. Other features (multiple equilibria, centennial oscillations) occur already in two-dimensional models, some of which have only a frictional/hydrostatic momentum balance [8, 12, 42, 44]. However, certainly the strength of the flow is dependent on the viscosity and hence quantitative changes may occur in the bifurcation diagrams. Hence, it is expected that lowering the horizontal viscosity will introduce shifts in bifurcation points but that no new flow regimes of ocean circulation regimes will appear.

To summarize, while the techniques are applied here only to a relatively simple model, the numerical methods seem capable of attacking the problems of the physics of multiple equilibria and low-frequency ((inter)decadal to centennial time scale) oscillations of the large-scale ocean circulation systematically. This is important for understanding the factors which control stability and variability of the thermohaline ocean circulation.

\section{ACKNOWLEDGMENTS}

This work was supported by the Netherlands Organization for Scientific Research (NWO) under a PIONIER grant to the first author. Use of the computing facilities was sponsored by the National Computing Facilities Foundation (NCF) with financial support from NWO. We thank Lianke te Raa and Wilbert Weijer for their contributions to the development of the model presented here.

\section{REFERENCES}

1. E. F. F. Botta, K. Dekker, Y. Notay, A. van der Ploeg, C. Vuik, F. W. Wubs, and P. M. de Zeeuw, How fast the Laplace equation was solved in 1995, Appl. Numer. Math. 24, 439 (1997).

2. E. F. F. Botta and F. W. Wubs, Matrix Renumbering ILU: An effective algebraic multilevel ILU-preconditioner for sparse matrices, SIAM J. Matrix Anal. Appl. 20(4), 1007 (1999).

3. E. F. F. Botta, F. W. Wubs, and A. van der Ploeg, A fast linear-system solver for large unstructured problems on a shared-memory computer, in Proceedings of the Conference on Algebraic Multilevel Iteration Methods with 
Applications, edited by O. Axelsson and B. Polman (University of Nijmegen, Nijmegen, The Netherlands, 1996), pp. 105-116.

4. W. S. Broecker, The great ocean conveyor, Oceanography 4, 79 (1991).

5. F. O. Bryan, High-latitude salinity effects and interhemispheric thermohaline circulations, Nature 323, 301 (1986).

6. K. Bryan, Accelerating the convergence to equilibrium of ocean-climate models, J. Phys. Oceanogr. 14, 666 (1984).

7. P. Cessi and G. R. Ierley, Symmetry-breaking multiple equilibria in quasi-geostrophic, wind-driven flows, J. Phys. Oceanography 25, 1196 (1995).

8. P. Cessi and W. R. Young, Multiple equilibria in two-dimensional thermohaline circulation, J. Fluid Mech. 241, 291 (1992).

9. K. N. Christodoulou and L. E. Scriven, Finding leading modes of a viscous free surface flow: An asymmetric generalized eigenproblem, J. Sci. Comput. 3, 355 (1988).

10. H. A. Dijkstra, Surface tension driven cellular patterns in three-dimensional boxes-part II: A bifurcation study, Microgravity Sci. Technol. 24, 415 (1995).

11. H. A. Dijkstra, Nonlinear Physical Oceanography (Kluwer Academic, Dordrecht, 2000).

12. H. A. Dijkstra and M. J. Molemaker, Symmetry breaking and overturning oscillations in thermohaline-driven flows, J. Fluid Mech. 331, 195 (1997).

13. H. A. Dijkstra, M. J. Molemaker, A van der Ploeg, and E. F. F. Botta, An efficient code to compute nonparallel flows and their linear stability, Comput. Fluids 24, 415 (1995).

14. J. K. Dukowicz and R. D. Smith, Implicit free-surface method for the Bryan-Cox-Semtner ocean model, J. Geophys. Res. 99, 7991 (1994).

15. P. R. Gent and J. C. McWilliams, Isopycnal mixing in ocean circulation models, J. Phys. Oceanogr. 20, 150 (1990).

16. I. Goldhirsch, S. A. Orszag, and B. K. Maulik, An efficient method for computing leading eigenvalues and eigenvectors of large asymmetric matrices. J. Sci. Comput. 2, 33 (1987).

17. G. H. Golub and C. F. Van Loan, Matrix Computations, 3rd ed. (Johns Hopkins Press, Baltimore, 1996).

18. R. J. Greatbatch and S. Zhang, An interdecadal oscillation in an idealized ocean basin forced by constant heat flux, J. Climate 8, 82 (1995).

19. I. Gustafsson, A class of first order factorization methods, BIT 18, 142 (1978).

20. R. L. Haney, Surface thermal boundary conditions for ocean circulation models, J. Phys. Oceanogr. 4, 241 (1971).

21. T. Huck, A. Colin de Verdiere, and A. J. Weaver, Interdecadal variability of the thermohaline circulation in box-ocean models forced by fixed surface fluxes, J. Phys. Oceanogr. 29, 865 (1999).

22. H. B. Keller, Numerical solution of bifurcation and nonlinear eigenvalue problems, in Applications of Bifurcation Theory, edited by P. H. Rabinowitz (Academic Press, New York, 1977).

23. P. D. Killworth, A two-level wind and buoyancy driven thermocline model, J. Phys. Oceanogr. 15, 1414 (1985).

24. W. G. Large, J. C. McWilliams, and S. C. Doney, Oceanic vertical mixing: A review and a model with nonlocal boundary layer parameters, Rev. Geophys. 32, 363 (1994).

25. E. Maier-Reimer, U. Mikolajewicz, and K. Hasselman, Mean circulation of the Hamburg LSG OGCM and its sensitivity to the thermohaline surface forcing, J. Phys. Oceanogr. 23, 731 (1993).

26. S. Manabe and R. J. Stouffer, Two stable equilibria of a coupled ocean-atmosphere model, J. Climate 1, 841 (1988).

27. S. Manabe and R. J. Stouffer, Century-scale effects of increased $\mathrm{CO}_{2}$ on the ocean-atmosphere system, Nature 364, 215 (1993).

28. J. Marotzke, Instabilities and multiple steady states of the thermohaline circulation, in Ocean Models in Climate Problems, edited by D. L. T. Anderson and J. Willebrand (Kluwer Academic, Dordrecht/Norwell MA, 1989), pp. 501-511.

29. J. Marotzke, P. Welander, and J. Willebrand, Instability and multiple steady states in a meridional-plane model of thermohaline circulation, Tellus 40, 162 (1988). 
30. A. Meijster and F. W. Wubs, Towards an implementation of a multilevel ILU preconditioner on sharedmemory computers, in Lecture Notes in Computer Science 1823 (Springer-Verlag, Berlin/New York, 2000), pp. 109-118.

31. C. Quon and M. Ghil, Multiple equilibria in thermosolutal convection due to salt-flux boundary conditions, J. Fluid Mech. 245, 449 (1992).

32. S. Rahmstorf, A fast and complete convection scheme for ocean models, Ocean Modelling 101, 9 (1993).

33. Y. Saad, Variations on Arnoldi's method for computing eigenelements of large unsymmetric matrices, Lin. Alg. Appl. 34, 269 (1980).

34. Y. Saad, Iterative Methods for Sparse Matrices (PWS-Kent, Boston, 1996).

35. M. J. Schmeits and H. A. Dijkstra, On the physics of the 9 months variability in the Gulf Stream region: Combining data and dynamical systems analysis, J. Phys. Oceanogr. 30, 1967 (2000).

36. W. J. Schmitz, On the interbasin-scale thermohaline circulation, Rev. Geophys. 33, 151 (1995).

37. G. L. G. Sleijpen and H. A. Van der Vorst, A Jacobi-Davidson iteration method for linear eigenvalue problems, SIAM J. Matrix Anal. Appl. 17, 410 (1996).

38. W. J. Steward and A. Jennings, A simultaneous iteration algorithm for real matrices, ACM Trans. Math. Software 7, 184 (1981).

39. H. Stommel, Thermohaline convection with two stable regimes of flow, Tellus 2, 244 (1961).

40. L. A. Te Raa and H. A. Dijkstra, Instability of the thermohaline ocean circulation on interdecadal time scales, J. Phys. Oceanogr., in press (2002).

41. S. F. B. Tett, T. C. Johns, and J. F. B Mitchell, Global and regional variability in a coupled AOGCM, Climate Dyn. 13, 303 (1997).

42. O. Thual and J. C. McWilliams, The catastrophe structure of thermohaline convection in a two-dimensional fluid model and a comparison with low-order box models, Geophys. Astrophys. Fluid Dyn. 64, 67 (1992).

43. J. J. Van Dorsselaer, Computing eigenvalues occurring in continuation methods with the Jacobi-Davidson QZ method, J. Comput. Phys. 138, 714 (1997).

44. M. Vellinga, Instability of two-dimensional thermohaline circulation, J. Phys. Oceanogr. 26, 305 (1996).

45. A. Weaver, J. Marotzke, P. F. Cummings, and E. S. Sarachik, Stability and variability of the thermohaline circulation, J. Phys. Oceanogr. 23, 39 (1993).

46. A. J. Weaver and T. M. Hughes, On the incompatibility of ocean and atmosphere and the need for flux adjustments, Climate Dyn. 12, 141 (1996).

47. W. Weijer and H. A. Dijkstra, Bifurcations of the three-dimensional thermohaline circulation: the double hemispheric case, J. Mar. Res., in press.

48. P. Welander, Thermohaline effects in the ocean circulation and related simple models, in Large Scale Transport Processes in Oceans and Atmosphere, edited by J. Willebrand and D. L. T. Anderson (Reidel, Dordrecht, 1986), pp. 163-200.

49. M. Winton, The role of horizontal boundaries in parameter sensitivity and decadal-scale variability of coarseresolution ocean general circulation models, J. Phys. Oceanogr. 26, 289 (1996).

50. M. Winton and E. S. Sarachik, Thermohaline oscillations induced by strong steady salinity forcing of ocean general circulation models, J. Phys. Oceanogr. 23, 1389 (1993).

51. R. A. Wood, A. B. Keen, J. F. B. Mitchell, and J. M. Gregory, Changing spatial structure of the thermohaline circulation in response to atmospheric $\mathrm{CO}_{2}$ forcing in a climate model, Nature 399, 572 (1999).

52. D. G. Wright and T. F. Stocker, A zonally averaged model for the thermohaline circulation, Part I: Model development and flow dynamics, J. Phys. Oceanogr. 21, 1713 (1991).

53. F. L. Yin and E. S. Sarachik, An efficient convective adjustment scheme for ocean general circulation models, J. Phys. Oceanogr. 24, 1425 (1994). 